\title{
Preliminary Health Risk Assessment of Water from Asa River, Ilorin, North-Central Nigeria
}

${ }^{1}$ Omotoso, O. A.; ${ }^{2}$ Ojo O. J.; ${ }^{3}$ Ige, O.O.; ${ }^{4}$ Ibrahim, K. O.; ${ }^{5}$ Yusuf, M. A.; ${ }^{6}$ Olasehinde, A. A.; ${ }^{7}$ Olelewe, M. C. and ${ }^{8}$ Adewoye, O.A.

1,3,4,5,6,7 Department of Geology and Mineral Sciences; University of Ilorin, Ilorin, Nigeria;

${ }^{2}$ Department of Geology, Federal University Oye-Ekiti, Nigeria

${ }^{8}$ Department of Earth Science, Ladoke Akintola University of Technology, Ogbomoso, Nigeria

Corresponding Author E-mail: omotoso.oa@ unilorin.edu.ng Tel: +2348067780267

Submitted on: 02/10/2020;

Accepted on: 18/01/2021

\begin{abstract}
This study was carried out to establish health impacts of some trace elements (Al, As, $B a, C r, C u$, $\mathrm{Fe}, \mathrm{Mn}, \mathrm{Ni}, \mathrm{Pb}$ and $\mathrm{Zn}$ ) in Asa-river, using Inductively Coupled Plasma-Mass Spectrometer technique for the chemical analysis. Laboratory results show that Al, Fe and Mn average concentrations are higher than prescribed limits unlike others. The Contamination Indexes reveal that the elements are heavily loaded in the water. The exposure dose in both adults and children ranges from 0.021 in As to 181 in Fe (in adults) and from 0.068 in As to 576 in Fe (in children). The average Hazard Quotient (HQ) values for adults and children are generally lower than one except Mn that has average value greater than one in both age groups. However, for children, individual $H Q$ in Fe in some of the locations are >1. The Hazard Index (HI)for adults and children ranged from low to high. The average values of Chronic Daily Intake (CDI) are generally $<1$. However, in children, values for Fe in some locations are >1. The average Carcinogenic Risk $(\mathrm{CR})$ of $\mathrm{Cr}$ and $\mathrm{Pb}$ computed for adults and children indicated that the values are greater than the risk limit of $10^{-6}$ and $10^{-4}$. This could result in potential health risk to the consumers. The sources of these trace elements are largely from various anthropogenic activities and slightly from weathering of rocks in the study area. It is recommended that adequate and well monitored precautions need to be taken to safeguard the health of the consumers especially the children with low immunity.
\end{abstract}

Keynotes: Health Risk Assessment, Asa River, Hazard Index, Chronic Daily Intake, Carcinogenic Risk.

\section{Introduction}

The ravaging of various diseases across the globe in recent times calls for global consideration and intervention because millions of people are being plagued and this has geometrically increased the mortality rate. In developing world like Nigeria there are also problems of indiscriminate disposal of wastes (industrial wastes, agricultural wastes, domestic wastes, municipal wastes) and this scenario cuts across the rural and urban areas of the country. These waste materials find their ways into near or faraway streams, rivers and groundwater, thereby increasing the elemental compositions of toxic elements in the consumable water phases (Hynds et al., 2014; Lindgren et al., 2016; Edokpayi et al., 2018). This directly or indirectly affects the quality of the sources of water and ultimately impairs the health of the consumers.

Similar works have been carried out across the globe by several researchers. Edokpayi et al., 2018 evaluated water quality and human risk assessment due to heavy metals in groundwater around Muledane area of Vhembe District, Limpopo Province, South Africa. Inductively Coupled Plasma Optical Atomic Spectrophotometer (ICP-OES) was used to analyze the water samples. The results of their study revealed that ingestion of the investigated boreholes water poses carcinogenic risk 
regarding the estimated $\mathrm{Mn}, \mathrm{Fe}$ and $\mathrm{Cu}$ for adults and children after Quantitative Health Risk Assessment of the water.

The study area is underlain by the basement complex of south western part of Nigeria, which is of Precambrian to lower Paleozoic in age (Rahaman, 1976; Oluyide, 1979). This consists of gneisses and migmatites, metasediments (schists, quartzites and metavolcanics and older Pan-African granite and late-stage minor pegmatitic and aplitic intrusives (Oluyide et al., 1998; Omotoso et. al., 2011). According to the work of Olasehinde et al. (1998), Ilorin is situated on the undifferentiated Precambrian Basement Complex rocks of granitic and metamorphic origin. It is also reported that the oldest rocks in the area comprise gneiss complex whose principal member is biotite-hornblende gneiss with intercalated amphibolites and other rock types in the area are the older granite mainly porphyritic granite, gneiss and granite-gneiss and quartz schist.

Asa river is one of the major sources of drinking water supply in Ilorin metropolis which was dammed around Asa township of Ilorin city. The water supplies industrial, agricultural and domestic sectors of the city. This same water resources serves as a source of irrigation for the farming system in the area. It also serves as a source of water for Fish ponds in the area. Consequently, as a result of various anthropogenic and natural weathering of rocks in the area, there is the need to critically examine the qualitative and quantitative health risks of the water resources so as to safeguard the health of the consumers.

\section{Methodology}

Environmental Settings of the Study Area

According to NOAA, 2016, the study area is situated in the savannah region of Nigeria which also lies within longitudes $4^{\circ} 30^{\prime} \mathrm{E}, 4^{\circ} 40^{\prime} \mathrm{E}$ and latitudes $8^{\circ} 19^{\prime} \mathrm{N}, 8^{\circ} 29^{\prime} \mathrm{N}$. The weather condition in the region is of two main types namely rainy season and dry season. The rainy season starts in the month of late March and ends around October, while dry season begins in November and ends in early March. The average annual rainfall ranges between $4.6 \mathrm{~mm}$ in November and $211.1 \mathrm{~mm}$ in September while the average relative humidity ranges from $29 \%$ in February to $70.2 \%$ in August. The mean annual temperature falls within $27^{\circ} \mathrm{C}$ to $30^{\circ} \mathrm{C}$.

The location map of the study area is presented in Figure 1 and this also shows the sampling points.

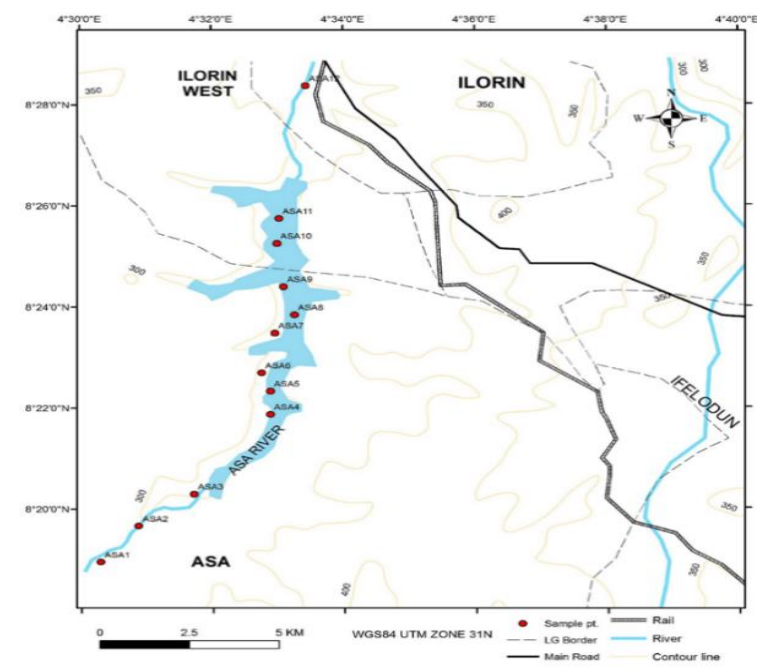

Figure 1: Location Map of the Study area showing Sampling Points (carved from topographical map sheet 223 NW, Ilorin, Nigeria, Published by Federal Survey, Nigeria, 1966) 
The study was carried out during the dry season (that is, January, 2017). Random sampling method was employed in the collection of the water samples. $60 \mathrm{ml}$ white bottle containers with white cover were used to collect the water samples. Topographical map sheet $223 \mathrm{NW}$, Ilorin was used as base map to navigate the sampling points with the aid of GPS. With the aid of the location map, 12 sampling points were randomly mapped out because of the challenge of accessibility in some areas which did not support systemic sampling technique or gridding.

At each proposed sampling point, water sample was taken randomly. Before sampling, the white bottle container was rinsed severally to remove every dirt from the container, then the containers were filled with the water samples to the brim respectively. Air space was prevented to make the water in the container airtight. Few drops of concentrated nitric acid were added to the water samples respectively and covered tightly immediately. Nitric acid prevents the precipitation of the cations from solution. After each sampling, the bottle was labelled accordingly. The samples were shipped to the laboratory for hydrochemical analysis. The water samples were analyzed in ACME laboratory, Vancouver, Canada, North America, using Inductively Coupled Plasma-Mass Spectrometer (ICP-MS) laboratory technique.

\section{Data Evaluation}

The selected trace elements ( $\mathrm{Al}, \mathrm{As}, \mathrm{Ba}, \mathrm{Cr}, \mathrm{Cu}, \mathrm{Fe}, \mathrm{Mn}, \mathrm{Ni}, \mathrm{Pb}$ and $\mathrm{Zn}$ ) were examined by: comparing their respective concentrations with WHO (2006) and NSDWQ (2007); determining some of their contamination indexes (i.e. contamination factor and degree of contamination); health risk assessment using the average daily dose, hazard quotient, hazard index, chronic daily intake and carcinogenic risk. The WHO standard, Nigerian standard for drinking water qualities and the Mean Composition of World Rivers (MCWR) were presented in Table 1 respectively. The MCWR was adopted to normalize the results of the Contamination Indexes.

Table 1: WHO and Nigerian Standards for Drinking Water Quality for potable water and mean composition of world rivers.

\begin{tabular}{lccc}
\hline $\begin{array}{l}\text { Trace } \\
\text { Elements }\end{array}$ & $\begin{array}{l}\text { WHO } \\
\text { Standard } \\
\text { (ppm), 2006 }\end{array}$ & $\begin{array}{l}\text { NSDWQ, } \\
\text { 2007 (ppm) }\end{array}$ & $\begin{array}{c}\text { Mean composition of } \\
\text { world rivers in ppm, } \\
\text { (after Viers et al., 2009) }\end{array}$ \\
\hline $\begin{array}{l}\text { Aluminum, } \\
\text { Al }\end{array}$ & 0.2 & 0.2 & 0.32 \\
Arsenic, As & 0.01 & 0.01 & 0.00062 \\
Barium, Ba & 0.3 & 0.7 & 0.023 \\
Chromium, & 0.05 & 0.05 & 0.0007 \\
Cr & 2 & 1 & 0.00148 \\
Copper, Cu & 0.3 & 0.3 & 0.066 \\
Iron, Fe & 0.5 & 0.2 & 0.00042 \\
$\begin{array}{l}\text { Manganese, } \\
\text { Mn }\end{array}$ & 0.02 & 0.001 & 0.0008 \\
Nickel, Ni & 3 & 0.01 & 0.00008 \\
Lead, Pb & 0.003 & 0.003 & 0.0006 \\
Cadmium, & Cd & 0.03 & $2009)$ \\
\hline
\end{tabular}

(WHO, 2006; NSDWQ, 2007; Vier et al., 2009) 
Contamination Factor: This can be expressed as the single index usually determined using equation 1:

$$
C f=\frac{C m}{B m}
$$

$\mathrm{C} f$ is the contamination factor of the trace element of interest; $\mathrm{Cm}$ is the concentration of the trace element in the sample; Bm is the background concentration of the trace element in the sample. MCWR was used for the value of Bm in this research. Atiemo et al., 2011 presented four categories of contamination factors which include the following:

$<1=$ low contamination factor;

$1-3=$ moderate contamination factor;

3-6=considerable contamination factor;

$>6=$ very high contamination factor

Degree of Contamination $\left(\mathbf{C}_{\mathbf{d e g}}\right)$ : This is the addition of all the contamination factors in the sample and it can be expressed as indicated in equation 2 :

$$
C_{d e g}=\sum^{c_{m}} / B_{m}
$$

$\mathrm{Cm}$ is the concentration of water; $\mathrm{Bm}$ is the local background concentration of trace element, $\mathrm{m}$ within the pristine area of catchment. Atiemo et al., 2011 also identified four categories namely: < $8=$ low degree of contamination, 8-16=moderate degree of contamination, $16-32=$ considerable degree of contamination and $>32=$ very high degree of contamination.

\section{Quantitative Health Risk Assessment:}

The Average Daily Dose: The Average Daily Dose was computed using equation 3 shown below to estimate the health risk of the water samples from the study area.

$$
A D D=\frac{C w a t e r * I R * E F * E D}{B W * A T}
$$

where, ADD is the Average Daily Dose similar to the exposure dose through ingestion of water ( $\mu \mathrm{g} / \mathrm{kg} /$ day); $\mathrm{C}_{\text {water }}$ is the average concentration of the estimated metals in water $(\mu \mathrm{g} / \mathrm{L})$; IR is the ingestion rate in this research $(2.72 \mathrm{~L} /$ day for adults; $1.8 \mathrm{~L}$ /day for children); $\mathrm{EF}$ is the exposure frequency (365 days/year); ED is the exposure duration (70 years for adults; and 6 years for children); BW is the average body weight (70 kg for adults; $15 \mathrm{~kg}$ for children); AT is the averaging time (365 days/year $\times 70$ years for an adult; 365 days/year $\times 6$ years for a child), Edokpayi, et al. (2018). Hazard Quotient (HQ): The non-carcinogenic health risk as a result of the exposure to surface water resources consumption was computed as the hazard quotient (HQ). Equation 4 is used to calculate the HQ of Asa river:

$$
H Q=\frac{A D D}{R f D}
$$

$\mathrm{ADD}$ is the average daily dose similar to the exposure dose through ingestion of water ( $\mu \mathrm{g} / \mathrm{kg} / \mathrm{day})$; $\mathrm{R}_{\mathrm{f}} \mathrm{D}$ is the reference dose of a specific element. The reference dose for $\mathrm{Al}, \mathrm{As}, \mathrm{Ba}, \mathrm{Cr}, \mathrm{Cu}, \mathrm{Fe}, \mathrm{Mn}$, $\mathrm{Ni}, \mathrm{Pb}$ and $\mathrm{Zn}$ are: $1 \mathrm{mg} / \mathrm{kg} /$ day, $0.0003 \mathrm{mg} / \mathrm{kg} /$ day, $0.2 \mathrm{mg} / \mathrm{kg} / \mathrm{day}, 1.5 \mathrm{mg} / \mathrm{kg} /$ day, $0.04 \mathrm{mg} / \mathrm{kg} / \mathrm{day}$, $0.7 \mathrm{mg} / \mathrm{kg} /$ day, $0.046 \mathrm{mg} / \mathrm{kg} /$ day, $0.02 \mathrm{mg} / \mathrm{kg} / \mathrm{day}, 0.0035 \mathrm{mg} / \mathrm{kg} / \mathrm{day}$ and $0.3 \mathrm{mg} / \mathrm{kg} / \mathrm{day}$ respectively (USEPA, 2001). If the value of HQ is greater than unity, that is, 1 , there is possibility of non-carcinogenic negative effects on health while HQ value less than unity infers that the 
LAUTECH Journal of Civil and Environmental Studies

Volume 6, Issue 1; March 2021

exposure to the surface water consumption would not likely have any practical effect on the consumers (USEPA 2001, Yuan et al., 2017; Maxwell et al., 2018; Joel et al., 2018).

Hazard Index (HI): To assess the overall potential non-carcinogenic health effects posed by more than one metal and pathway, the addition of the computed HQs across metals/trace elements was expressed as hazard index (HI) using the equation 5 according to USEPA (1989):

$$
H I=\sum_{i=1}^{n} H Q
$$

$\mathrm{HI}>1$ is an indication of a potential adverse effect on human health (Li SY and Zhang, 2010; Naveedullah et al., 2014). Table 2 presents the classification of non-carcinogenic risk.

Table 2: Classification of non-carcinogenic risk

\begin{tabular}{lll}
\hline Risk Level & Hazard Index $(\mathrm{HI})$ & Chronic Risk \\
\hline 1 & $<0.1$ & Negligible \\
2 & $\geq 0.1<1$ & Low \\
3 & $\geq 1<4$ & Medium \\
4 & $\geq 4$ & High \\
\hline & & (USEPA, 1989)
\end{tabular}

Chronic Daily Intake (CDI): Edokpayi et al (2018) calculated Chronic daily intake (CDI) of trace elements through ingestion using equation 6 below:

$$
C D I=C_{\text {water }} * \frac{D I}{B W}
$$

where $\mathrm{C}_{\text {water, }}$ DI and BW denote the concentration of trace elements in water (measured in $\mathrm{mg} / \mathrm{kg}$ ), average daily intake of water $(2.72 \mathrm{~L} /$ day for adults; $1.8 \mathrm{~L} /$ day for children) and body weight $(70 \mathrm{~kg}$ for adults; $15 \mathrm{~kg}$ for children), respectively.

Carcinogenic Risk (CR): Edokpayi et al (2018) also used equation 7 below to estimate the carcinogenic risk (CR) through ingestion and this was also adopted in this research:

$$
C R_{\text {ing }}=\frac{\text { Exping }}{S F_{\text {ing }}}
$$

where, $\mathrm{CR}_{\text {ing }}=$ carcinogenic risk via ingestion route and $\mathrm{SF}_{\text {ing }}=$ carcinogenic slope factor where $\mathrm{Pb}$ is $8.5 \mathrm{E}, \mathrm{Cd}$ is $6.1 \mathrm{E}+03$ and $\mathrm{Cr}$ is $5.0 \mathrm{E}+02 \mu \mathrm{g} / \mathrm{kg} /$ day (Iqbal and Shah, 2013; Naveedullah et al., 2014; Asare-Donkor et al., 2016). The $\mathrm{CR}_{\text {ing }}$ values for other trace elements were not computed in this study because of unobtainability of the $\mathrm{SF}_{\text {ing }}$ values.

\section{Results and Discussion}

\section{Hydrochemical results}

The results of some of the trace elements in the samples analyzed are presented in Table 3 and Figure 2 illustrates the profile of the average concentrations of the selected trace elements compared with WHO (2006) and NSDWQ (2007. Fe ranges from 0.58 to $15.02 \mathrm{ppm}$ (average=4.80ppm), Mn ranges from 0.048 to $6.256 \mathrm{ppm}$ (average $=1.61 \mathrm{ppm}$ ), Al ranges from 0.06 to $4.24 \mathrm{ppm}$ (average $=0.79$ ), Ba ranges from 0.057 to $0.406 \mathrm{ppm}$ (average $=0.155 \mathrm{ppm}), \mathrm{Zn}$ ranges from 0.004 to $0.0441 \mathrm{ppm}$ (average $=0.011 \mathrm{ppm}), \mathrm{Cu}$ ranges from 0.0015 to $0.023 \mathrm{ppm}$ (average $=0.006 \mathrm{ppm}), \mathrm{Pb}$ ranges from 0.0017 to $0.0193 \mathrm{ppm}$ (average $=0.0043$ ), $\mathrm{Ni}$ ranges from 0.0015 to $0.0078 \mathrm{ppm}$ (average $=0.0026 \mathrm{ppm}), \mathrm{Cr}$ ranges from 0.0004 to $0.0072 \mathrm{ppm}($ average $=0.0021 \mathrm{ppm})$ and As ranges 
from 0.0004 to $0.0011 \mathrm{ppm}$. Based on average values, their concentration sequence is as follows: $\mathrm{Fe}>\mathrm{Mn}>\mathrm{Al}>\mathrm{Ba}>\mathrm{Zn}>\mathrm{Cu}>\mathrm{Pb}>\mathrm{Ni}>\mathrm{Cr}>\mathrm{As}$.

Table 3: Hydrochemical Analysis Data of Selected Trace Elements in Asa River and their Statistical Summary

\begin{tabular}{|c|c|c|c|c|c|c|c|c|c|c|}
\hline & \multicolumn{10}{|c|}{ Trace Elements (ppm) } \\
\hline & Al & As & $\mathrm{Ba}$ & $\mathrm{Cr}$ & $\mathrm{Cu}$ & $\mathrm{Fe}$ & $M n$ & $\mathbf{N i}$ & $\mathrm{Pb}$ & Zn \\
\hline WHO, 2006 & 0.2 & 0.01 & 0.3 & 0.05 & 2 & 0.3 & 0.5 & 0.02 & 0.01 & 3 \\
\hline \multicolumn{11}{|l|}{ NSDW, } \\
\hline \multirow[t]{2}{*}{2007} & 0.2 & 0.01 & 0.7 & 0.05 & 1 & 0.3 & 0.2 & 0.001 & 0.01 & 3 \\
\hline & & 0.000 & 0.1459 & 0.001 & 0.002 & & 1.3190 & 0.002 & 0.002 & 0.004 \\
\hline \multirow[t]{2}{*}{ ASA-1 } & 0.145 & 4 & 3 & 1 & 5 & 0.582 & 8 & 2 & 1 & 8 \\
\hline & & 0.000 & 0.1679 & 0.001 & 0.009 & & 3.8232 & 0.002 & 0.001 & 0.016 \\
\hline \multirow[t]{2}{*}{ ASA-2 } & 0.173 & 6 & 8 & 2 & 8 & 1.335 & 4 & 3 & 7 & 5 \\
\hline & & 0.001 & 0.2774 & 0.001 & 0.001 & & & 0.001 & 0.004 & \\
\hline \multirow[t]{2}{*}{ ASA-3 } & 0.132 & 1 & 3 & 1 & 8 & 10.092 & 6.256 & 5 & 7 & 0.004 \\
\hline & & & 0.4060 & 0.007 & & & & 0.007 & 0.019 & 0.044 \\
\hline \multirow[t]{2}{*}{ ASA-4 } & 4.241 & 0.001 & 1 & 2 & 0.023 & 12.943 & 2.7234 & 8 & 3 & 1 \\
\hline & & 0.000 & 0.1108 & 0.001 & 0.003 & & 1.0589 & 0.002 & 0.002 & 0.005 \\
\hline \multirow[t]{2}{*}{ ASA-5 } & 0.633 & 6 & 3 & 8 & 5 & 6.639 & 4 & 4 & 3 & 3 \\
\hline & & 0.000 & & 0.001 & 0.004 & & 0.3017 & 0.001 & 0.005 & 0.011 \\
\hline \multirow[t]{2}{*}{ ASA-6 } & 0.785 & 4 & 0.1137 & 3 & 1 & 2.864 & 5 & 7 & 3 & 1 \\
\hline & & 0.000 & 0.1116 & 0.002 & 0.011 & & 0.2349 & 0.002 & 0.005 & 0.012 \\
\hline \multirow[t]{2}{*}{ ASA-7 } & 1.709 & 4 & 3 & 9 & 1 & 3.273 & 8 & 9 & 5 & 5 \\
\hline & & 0.000 & 0.0708 & 0.003 & 0.005 & & 0.0803 & 0.002 & 0.002 & 0.005 \\
\hline \multirow[t]{2}{*}{ ASA-8 } & 0.855 & 4 & 9 & 3 & 6 & 1.676 & 8 & 4 & 4 & 1 \\
\hline & & 0.000 & 0.2137 & 0.002 & 0.002 & & 2.1818 & 0.002 & 0.002 & 0.004 \\
\hline \multirow[t]{2}{*}{ ASA-9 } & 0.314 & 7 & 4 & 1 & 5 & 15.02 & 4 & 2 & 2 & 3 \\
\hline & & 0.000 & 0.1093 & 0.001 & 0.003 & & 0.8111 & 0.001 & 0.002 & \\
\hline \multirow[t]{2}{*}{ ASA-10 } & 0.36 & 4 & 8 & 7 & 4 & 1.726 & 5 & 8 & 2 & 0.009 \\
\hline & & 0.000 & 0.0572 & 0.000 & 0.003 & & 0.0478 & 0.002 & 0.001 & 0.008 \\
\hline \multirow[t]{2}{*}{ ASA-11 } & 0.09 & 4 & 5 & 8 & 6 & 0.693 & 9 & 1 & 9 & 8 \\
\hline & & 0.000 & 0.0796 & 0.000 & 0.001 & & 0.4917 & & 0.001 & 0.005 \\
\hline \multirow[t]{2}{*}{ ASA-12 } & 0.06 & 4 & 1 & 4 & 5 & 0.769 & 2 & 0.002 & 7 & 7 \\
\hline & 0.791 & 0.000 & & 0.002 & 0.006 & & & 0.002 & 0.004 & 0.010 \\
\hline \multirow[t]{2}{*}{ average } & 4 & 6 & 0.1554 & 1 & 0 & 4.8010 & 1.6109 & 6 & 3 & 9 \\
\hline & 0.060 & 0.000 & & 0.000 & 0.001 & & & 0.001 & 0.001 & 0.004 \\
\hline \multirow[t]{2}{*}{$\min }$. & 0 & 4 & 0.0573 & 4 & 5 & 0.5820 & 0.0479 & 5 & 7 & 0 \\
\hline & 4.241 & 0.001 & & 0.007 & 0.023 & 15.020 & & 0.007 & 0.019 & 0.044 \\
\hline \multirow[t]{2}{*}{$\max }$. & 0 & 1 & 0.4060 & 2 & 0 & 0 & 6.2560 & 8 & 3 & 1 \\
\hline & 1.185 & 0.000 & & 0.001 & 0.006 & & & 0.001 & 0.004 & 0.011 \\
\hline \multirow[t]{2}{*}{ stdev. } & 0 & 2 & 0.1007 & 8 & 1 & 5.1376 & 1.8759 & 7 & 9 & 1 \\
\hline & 1.404 & 0.000 & & 0.000 & 0.000 & 26.395 & & 0.000 & 0.000 & 0.000 \\
\hline \multirow[t]{2}{*}{ var. } & 3 & 0 & 0.0101 & 0 & 0 & 2 & 3.5190 & 0 & 0 & 1 \\
\hline & 0.337 & 0.000 & & 0.001 & 0.003 & & & 0.002 & 0.002 & 0.007 \\
\hline med. & 0 & 4 & 0.1127 & 5 & 6 & 2.2950 & 0.9350 & 2 & 3 & 3 \\
\hline
\end{tabular}


LAUTECH Journal of Civil and Environmental Studies

Volume 6, Issue 1; March 2021

Based on the average concentrations computed, it was discovered that $\mathrm{Al}, \mathrm{Fe}$ and $\mathrm{Mn}$ are above the recommended values of 0.2, 0.3 and $0.5 \mathrm{ppm}$ of WHO (2006) and NSDWQ (2007) respectively while others are quite below the prescribed values. These three elements are of health significant which could hamper human health leading to shortage of the consumers' life span. It has been reported that excess iron in water constitute health hazard to consumers. It leads to gene mutation resulting in haemochromatosis whose symptoms include fatigue, weight loss, joint pains and ultimate heart disease, liver problems and diabetes (Ekere et al., 2014). In addition, excess Fe in water also leads to severe allergic reaction (e.g. rashes and itching), breathing difficulty, tightness in chest, swelling of the mouth, lips and face, black tarry stools as well as blood or streaks of blood in the stool including severe vomiting or stomach pain (Ekere et al., 2014). The elevated values could be as a result of agricultural practices (fish ponding, growing of crops using fertilizers, pesticides and herbicides) carrying out in the study area. It can be suggested that weathering of aluminosilicate minerals in the rocks and sediments from the area can contribute to elemental compositions of the water (Omotoso et al, 2018).

\section{Computed Contamination Indexes of $\mathrm{Al}, \mathrm{Fe}$ and Mn:}

The contamination indexes of the three trace elements were computed to support the sources of the elevated values in the water (Table 4). Figures 3 and 4 present the profiles of the contamination indexes for the average values of the three trace elements. The computed contamination factor of $\mathrm{Al}$ ranges from 0.19 to 13.25 , Fe ranges from 8.82 to 227.58 and $\mathrm{Mn}$ ranges from 114.02 to 14895 . Values of contamination factor greater than unity implies that the sources of ions are both from geogenic (weathering of rock materials in the area of study) and heavy anthropogenic factors while values less than unity indicate mainly from weathering of aluminosilicate minerals and rock materials in the area (Tijani, 2007, Omotoso et al., 2017; Omotoso 2018).

The Degree of contamination of $\mathrm{Al}, \mathrm{Fe}$ and $\mathrm{Mn}$ in the samples analyzed ranges from 124.81 in sample ASA 11 to 15048.56 in sample ASA 3. The average is 3910.61. This means that the samples are heavily contaminated with these elements/metals. Most especially according to Figure 5, ASA 2, ASA 3 and ASA 4 are the most heavily contaminated areas probably because of various agricultural practices in the area.

Table 4: Statistical Summary of Contamination Factors and Degree of Contamination of $\mathrm{Al}, \mathrm{Fe}$ and $\mathrm{Mn}$ in the sampled Water

\begin{tabular}{llllll}
\hline & \multicolumn{2}{l}{ Contamination Factor } & \multicolumn{2}{l}{ Degree of Contamination } \\
& Al & Fe & Mn & & \\
\hline average & 2.5 & 72.7 & 3835.4 & average & 3910.606 \\
min & 0.2 & 8.8 & 114.0 & min & 124.8051 \\
max & 13.3 & 227.6 & 14895.2 & max & 15048.56 \\
stdev & 3.7 & 77.8 & 4466.5 & stdev & 4507.29 \\
var & 13.7 & 6059.5 & 19949255.3 & var & 20315665 \\
med & 1.1 & 34.8 & 2226.3 & med & 2291.22 \\
\hline \hline
\end{tabular}

(after Tijani 2007; Omotoso and Ojo, 2017)

\section{Human Health Risk Assessment:}

Exposure dose through ingestion for adults: The computed statistical values of the exposure dose through ingestion for adults are presented in Table 5 and Figure 5 presents the profile of the exposure 
dose. On the average, $\mathrm{Al}, \mathrm{As}, \mathrm{Ba}, \mathrm{Cr}, \mathrm{Cu}, \mathrm{Fe}, \mathrm{Mn}, \mathrm{Ni}, \mathrm{Pb}$ and $\mathrm{Zn}$ have the following values respectively: $29.898 \mu \mathrm{g} / \mathrm{kg} /$ day, $0.02 \mu \mathrm{g} / \mathrm{kg} /$ day, $5.87 \mu \mathrm{g} / \mathrm{kg} /$ day, $0.078 \mu \mathrm{g} / \mathrm{kg} /$ day, $0.228 \mu \mathrm{g} / \mathrm{kg} / \mathrm{day}$, $181.37 \mu \mathrm{g} / \mathrm{kg} / \mathrm{day}, 60.855 \mu \mathrm{g} / \mathrm{kg} / \mathrm{day}, 0.099 \mu \mathrm{g} / \mathrm{kg} / \mathrm{day}, 0.162 \mu \mathrm{g} / \mathrm{kg} / \mathrm{day}$ and $0.413 \mu \mathrm{g} / \mathrm{kg} / \mathrm{day}$. The average values computed are greater than unity in $\mathrm{Al}, \mathrm{Ba}, \mathrm{Fe}$ and $\mathrm{Mn}$ while others are less than unity. Values below unity are generally recommended for a safe water quality. However, Al, Ba, Fe and $\mathrm{Mn}$ that has values higher than 1 are of health risk in adults' consumers.

Table 5: Statistical Summary of the Exposure Dose through Ingestion in Adults (in $\mu \mathrm{g} / \mathrm{kg} / \mathrm{day}$ ) Exposure Dose through Ingestion in Adults (Exping)

\begin{tabular}{|c|c|c|c|c|c|c|c|c|c|c|}
\hline $\begin{array}{l}\text { Sample } \\
\text { ID }\end{array}$ & $\mathrm{Al}$ & As & $\mathrm{Ba}$ & $\mathrm{Cr}$ & $\mathrm{Cu}$ & $\mathrm{Fe}$ & $\mathrm{Mn}$ & $\mathrm{Ni}$ & $\mathrm{Pb}$ & $\mathrm{Zn}$ \\
\hline ASA-1 & 5.478 & 0.015 & 5.513 & 0.042 & 0.094 & 21.987 & $\begin{array}{l}49.832 \\
144.43\end{array}$ & 0.083 & 0.079 & 0.181 \\
\hline ASA-2 & 6.536 & 0.023 & 6.346 & 0.045 & 0.370 & $\begin{array}{l}50.433 \\
381.25\end{array}$ & $\begin{array}{c}4 \\
236.33\end{array}$ & 0.087 & 0.064 & 0.623 \\
\hline ASA-3 & $\begin{array}{c}4.987 \\
160.21\end{array}$ & 0.042 & 10.481 & 0.042 & 0.068 & $\begin{array}{c}3 \\
488.95\end{array}$ & $\begin{array}{c}8 \\
102.88\end{array}$ & 0.057 & 0.178 & 0.151 \\
\hline ASA-4 & 6 & 0.038 & 15.338 & 0.272 & 0.869 & $\begin{array}{c}8 \\
250.80\end{array}$ & 4 & 0.295 & 0.729 & 1.666 \\
\hline ASA-5 & 23.913 & 0.023 & 4.187 & 0.068 & 0.132 & $\begin{array}{c}7 \\
108.19\end{array}$ & 40.004 & 0.091 & 0.087 & 0.200 \\
\hline ASA-6 & 29.656 & 0.015 & 4.295 & 0.049 & 0.155 & $\begin{array}{c}6 \\
123.64\end{array}$ & 11.399 & 0.064 & 0.200 & 0.419 \\
\hline ASA-7 & 64.562 & 0.015 & 4.217 & 0.110 & 0.419 & 7 & 8.877 & 0.110 & 0.208 & 0.472 \\
\hline ASA-8 & 32.300 & 0.015 & 2.678 & 0.125 & 0.212 & $\begin{array}{l}63.316 \\
567.42\end{array}$ & 3.037 & 0.091 & 0.091 & 0.193 \\
\hline ASA-9 & 11.862 & 0.026 & 8.075 & 0.079 & 0.094 & 2 & 82.425 & 0.083 & 0.083 & 0.162 \\
\hline ASA-10 & 13.600 & 0.015 & 4.132 & 0.064 & 0.128 & 65.204 & 30.643 & 0.068 & 0.083 & 0.340 \\
\hline ASA-11 & 3.400 & 0.015 & 2.163 & 0.030 & 0.136 & 26.180 & 1.809 & 0.079 & 0.072 & 0.332 \\
\hline ASA-12 & 2.267 & 0.015 & 3.007 & 0.015 & 0.057 & 29.051 & 18.576 & 0.076 & 0.064 & 0.215 \\
\hline averag & & 0.0214 & 5.8693 & 0.0783 & 0.2279 & 181.37 & 60.854 & 0.0985 & & 0.4130 \\
\hline e & 29.898 & 07 & 44 & 89 & 26 & 11 & 87 & 37 & 0.1615 & 37 \\
\hline & 2.2666 & 0.0151 & 2.1627 & 0.0151 & 0.0566 & 21.986 & 1.8091 & 0.0566 & 0.0642 & 0.1511 \\
\hline $\min$ & 67 & 11 & 78 & 11 & 67 & 67 & 78 & 67 & 22 & 11 \\
\hline & 160.21 & 0.0415 & 15.338 & & 0.8688 & 567.42 & 236.33 & 0.2946 & 0.7291 & \\
\hline $\max$ & 56 & 56 & 16 & 0.272 & 89 & 22 & 78 & 67 & 11 & 1.666 \\
\hline & 44.767 & 0.0094 & 3.8056 & 0.0686 & 0.2318 & 194.08 & 70.867 & 0.0633 & 0.1865 & 0.4207 \\
\hline stdev & 27 & 39 & 08 & 86 & 64 & 8 & 81 & 06 & 19 & 72 \\
\hline & 2004.1 & 8.91E- & 14.482 & 0.0047 & 0.0537 & 37670. & 5022.2 & 0.0040 & 0.0347 & 0.1770 \\
\hline var & 08 & 05 & 65 & 18 & 61 & 14 & 47 & 08 & 89 & 49 \\
\hline & 12.731 & 0.0151 & 4.2562 & 0.0566 & 0.1341 & & 35.323 & 0.0831 & & 0.2738 \\
\hline med & 11 & 11 & 33 & 67 & 11 & 86.7 & 92 & 11 & 0.085 & 89 \\
\hline
\end{tabular}

Exposure Dose through Ingestion for Children: The statistical values of the exposure dose through ingestion in children are presented in Table 6 and the profile is illustrated in Figure 6 . The average values for $\mathrm{Al}, \mathrm{As}, \mathrm{Ba}, \mathrm{Cr}, \mathrm{Cu}, \mathrm{Fe}, \mathrm{Mn}, \mathrm{Ni}, \mathrm{Pb}$ and $\mathrm{Zn}$ are: $94.97 \mu \mathrm{g} / \mathrm{kg} / \mathrm{day}, 0.068 \mu \mathrm{g} / \mathrm{kg} / \mathrm{day}$, $18.64 \mu \mathrm{g} / \mathrm{kg} / \mathrm{day}, 0.249 \mu \mathrm{g} / \mathrm{kg} / \mathrm{day}, 0.724 \mu \mathrm{g} / \mathrm{kg} / \mathrm{day}, 576.12 \mu \mathrm{g} / \mathrm{kg} / \mathrm{day}, 193.304 \mu \mathrm{g} / \mathrm{kg} / \mathrm{day}, 0.313$ $\mu \mathrm{g} / \mathrm{kg} / \mathrm{day}, 0.513 \mu \mathrm{g} / \mathrm{kg} / \mathrm{day}$ and $1.312 \mu \mathrm{g} / \mathrm{kg} /$ day respectively. The average values of $\mathrm{Al}, \mathrm{Ba}, \mathrm{Fe}$, 
LAUTECH Journal of Civil and Environmental Studies

Volume 6, Issue 1; March 2021

$\mathrm{Mn}$ and $\mathrm{Zn}$ are greater than unity while others are less than unity. The higher values indicate health risk in children which must not be overlooked.

Table 6: Statistical Summary of the Exposure Dose through Ingestion in Children (in $\mu \mathrm{g} / \mathrm{kg} / \mathrm{day})$

\begin{tabular}{|c|c|c|c|c|c|c|c|c|c|c|}
\hline \multirow{2}{*}{$\begin{array}{l}\text { Sample } \\
\text { ID }\end{array}$} & \multicolumn{10}{|c|}{ Exposure Dose through Ingestion in Children (Exping) } \\
\hline & $\mathrm{Al}$ & As & $\mathrm{Ba}$ & $\mathrm{Cr}$ & $\mathrm{Cu}$ & $\mathrm{Fe}$ & $\mathrm{Mn}$ & $\mathrm{Ni}$ & $\mathrm{Pb}$ & $\mathrm{Zn}$ \\
\hline \multirow{3}{*}{ ASA-1 } & & & & & & & 158.29 & & & \\
\hline & 17.400 & 0.048 & 17.512 & 0.132 & 0.300 & 69.840 & 0 & 0.264 & 0.252 & 0.576 \\
\hline & & & & & & 160.20 & 458.78 & & & \\
\hline \multirow[t]{2}{*}{ ASA-2 } & 20.760 & 0.072 & 20.158 & 0.144 & 1.176 & 0 & 9 & 0.276 & 0.204 & 1.980 \\
\hline & & & & & & 1211.0 & 750.72 & & & \\
\hline \multirow[t]{2}{*}{ ASA-3 } & 15.840 & 0.132 & 33.292 & 0.132 & 0.216 & 40 & 0 & 0.180 & 0.564 & 0.480 \\
\hline & 508.92 & & & & & 1553.1 & 326.80 & & & \\
\hline \multirow[t]{2}{*}{ ASA-4 } & 0 & 0.120 & 48.721 & 0.864 & 2.760 & 60 & 8 & 0.936 & 2.316 & 5.292 \\
\hline & & & & & & 796.68 & 127.07 & & & \\
\hline \multirow[t]{2}{*}{ ASA-5 } & 75.960 & 0.072 & 13.300 & 0.216 & 0.420 & 0 & 3 & 0.288 & 0.276 & 0.636 \\
\hline & & & & & & 343.68 & & & & \\
\hline \multirow[t]{2}{*}{ ASA-6 } & 94.200 & 0.048 & 13.644 & 0.156 & 0.492 & 0 & 36.210 & 0.204 & 0.636 & 1.332 \\
\hline & 205.08 & & & & & 392.76 & & & & \\
\hline \multirow[t]{2}{*}{ ASA-7 } & 0 & 0.048 & 13.396 & 0.348 & 1.332 & 0 & 28.198 & 0.348 & 0.660 & 1.500 \\
\hline & 102.60 & & & & & 201.12 & & & & \\
\hline \multirow[t]{2}{*}{ ASA-8 } & 0 & 0.048 & 8.507 & 0.396 & 0.672 & 0 & 9.646 & 0.288 & 0.288 & 0.612 \\
\hline & & & & & & 1802.4 & 261.82 & & & \\
\hline \multirow[t]{2}{*}{ ASA-9 } & 37.680 & 0.084 & 25.649 & 0.252 & 0.300 & 00 & 1 & 0.264 & 0.264 & 0.516 \\
\hline & & & & & & 207.12 & & & & \\
\hline ASA-10 & 43.200 & 0.048 & 13.126 & 0.204 & 0.408 & 0 & 97.338 & 0.216 & 0.264 & 1.080 \\
\hline ASA-11 & 10.800 & 0.048 & 6.870 & 0.096 & 0.432 & 83.160 & 5.747 & 0.252 & 0.228 & 1.056 \\
\hline ASA-12 & 7.200 & 0.048 & 9.553 & 0.048 & 0.180 & 92.280 & 59.006 & 0.240 & 0.204 & 0.684 \\
\hline \multicolumn{2}{|l|}{ averag } & & 18.643 & & & & 193.30 & & & \\
\hline e & 94.97 & 0.068 & 8 & 0.249 & 0.724 & 576.12 & 37 & 0.313 & 0.513 & 1.312 \\
\hline \multirow[t]{2}{*}{$\min$} & 7.2 & 0.048 & 6.87 & 0.048 & 0.18 & 69.84 & 5.7468 & 0.18 & 0.204 & 0.48 \\
\hline & & & 48.721 & & & & & & & \\
\hline \multirow[t]{2}{*}{$\max$} & 508.92 & 0.132 & 2 & 0.864 & 2.76 & 1802.4 & 750.72 & 0.936 & 2.316 & 5.292 \\
\hline & 142.20 & 0.0299 & 12.088 & 0.2181 & 0.7365 & 616.51 & 225.10 & 0.2010 & 0.5924 & 1.336 \\
\hline \multirow[t]{2}{*}{ stdev } & 19 & 82 & 4 & 78 & 08 & 47 & 95 & 89 & 72 & 57 \\
\hline & 20221. & 0.0008 & 146.12 & 0.0476 & 0.5424 & 38009 & 50674. & 0.0404 & 0.3510 & 1.786 \\
\hline \multirow[t]{2}{*}{ var } & 38 & 99 & 95 & 02 & 44 & 0.4 & 3 & 37 & 23 & 42 \\
\hline & & & 13.519 & & & & 112.20 & & & \\
\hline med & 40.44 & 0.048 & 8 & 0.18 & 0.426 & 275.4 & 54 & 0.264 & 0.27 & 0.87 \\
\hline
\end{tabular}

Hazard Quotient and Hazard Index for Adults: The computed hazard quotient, hazard index together with their statistical summary are presented in Table 7 and their graphical profiles are illustrated in Figures 7 and 8. The computed average values of hazard quotient for $\mathrm{Al}, \mathrm{As}, \mathrm{Ba}, \mathrm{Cr}$, $\mathrm{Cu}, \mathrm{Fe}, \mathrm{Mn}, \mathrm{Ni}, \mathrm{Pb}$ and $\mathrm{Zn}$ are: 0.0.299, 0.00153, 0.0293, 5.23E-05, 0.005698, 0.259, 1.32, 0.00493, 0.0461 and 0.00138 respectively. Only average value of $\mathrm{Mn}$ is greater than the recommended value of unity while others are less than unity (USEPA 1989; Su et al., 2017). 
However, the Hazard index ranges from 0.12 in sample ASA-12 to 5.798 in ASA-3 with an average of 1.7. That is, the range is from low to high (USEPA, 1989). This is an indication of health risk for the adults' consumers.

Table 7: Computed Hazard Quotient, Hazard Index and their Statistical Descriptions in Adults' Consumers

\begin{tabular}{|c|c|c|c|c|c|c|c|c|c|c|c|c|}
\hline \multicolumn{11}{|c|}{ Hazard Quotient (Adults) } & \multicolumn{2}{|c|}{ Hazard } \\
\hline & $\mathrm{Al}$ & As & $\mathrm{Ba}$ & $\mathrm{Cr}$ & $\mathrm{Cu}$ & $\mathrm{Fe}$ & $\mathrm{Mn}$ & $\mathrm{Ni}$ & $\mathrm{Pb}$ & $\mathrm{Zn}$ & & \\
\hline ASA- & 0.005 & 0.001 & 0.027 & $2.77 \mathrm{E}$ & 0.002 & 0.031 & 1.083 & 0.004 & 0.022 & 0.000 & ASA- & 1. \\
\hline 1 & 478 & 079 & 565 & -05 & 361 & 41 & 302 & 156 & 667 & 604 & 1 & 18 \\
\hline ASA- & 0.006 & 0.001 & 0.031 & $3.02 \mathrm{E}$ & 0.009 & 0.072 & 3.139 & 0.004 & 0.018 & 0.002 & ASA- & 3. \\
\hline 2 & 536 & 619 & 73 & -05 & 256 & 048 & 859 & 344 & 349 & 078 & 2 & 29 \\
\hline ASA- & 0.004 & 0.002 & 0.052 & 2.77E & 0.001 & 0.544 & 5.137 & 0.002 & 0.050 & 0.000 & ASA- & 5. \\
\hline 3 & 987 & 968 & 403 & -05 & 7 & 648 & 778 & 833 & 73 & 504 & 3 & 80 \\
\hline ASA- & 0.160 & 0.002 & 0.076 & 0.000 & 0.021 & 0.698 & 2.236 & 0.014 & 0.208 & 0.005 & ASA- & 3. \\
\hline 4 & 216 & 698 & 691 & 181 & 722 & 511 & 609 & 733 & 317 & 553 & 4 & 43 \\
\hline ASA- & 0.023 & 0.001 & 0.020 & $4.53 \mathrm{E}$ & 0.003 & 0.358 & 0.869 & 0.004 & 0.024 & 0.000 & ASA- & 1. \\
\hline 5 & 913 & 619 & 935 & -05 & 306 & 295 & 661 & 533 & 825 & 667 & 5 & 31 \\
\hline ASA- & 0.029 & 0.001 & 0.021 & $3.27 \mathrm{E}$ & 0.003 & 0.154 & 0.247 & 0.003 & 0.057 & 0.001 & ASA- & 0. \\
\hline 6 & 656 & 079 & 477 & -05 & 872 & 565 & 814 & 211 & 206 & 398 & 6 & 52 \\
\hline ASA- & 0.064 & 0.001 & 0.021 & 7.3E- & 0.010 & 0.176 & 0.192 & 0.005 & 0.059 & 0.001 & ASA- & 0. \\
\hline 7 & 562 & 079 & 086 & 05 & 483 & 638 & 979 & 478 & 365 & 574 & 7 & 53 \\
\hline ASA- & 0.032 & 0.001 & 0.013 & $8.31 \mathrm{E}$ & 0.005 & 0.090 & 0.066 & 0.004 & 0.025 & 0.000 & ASA- & 0. \\
\hline 8 & 3 & 079 & 39 & -05 & 289 & 451 & 013 & 533 & 905 & 642 & 8 & 24 \\
\hline ASA- & 0.011 & 0.001 & 0.040 & $5.29 E$ & 0.002 & 0.810 & 1.791 & 0.004 & 0.023 & 0.000 & ASA- & 2. \\
\hline 9 & 862 & 889 & 373 & -05 & 361 & 603 & 849 & 156 & 746 & 541 & 9 & 69 \\
\hline ASA- & 0.013 & 0.001 & 0.020 & $4.28 \mathrm{E}$ & 0.003 & 0.093 & 0.666 & 0.003 & 0.023 & 0.001 & ASA- & 0. \\
\hline 10 & 6 & 079 & 661 & -05 & 211 & 149 & 162 & 4 & 746 & 133 & 10 & 83 \\
\hline ASA- & 0.003 & 0.001 & 0.010 & $2.01 \mathrm{E}$ & 0.003 & 0.037 & 0.039 & 0.003 & 0.020 & 0.001 & ASA- & 0. \\
\hline 11 & 4 & 079 & 814 & -05 & 4 & 4 & 33 & 967 & 508 & 108 & & 12 \\
\hline ASA- & 0.002 & 0.001 & 0.015 & $1.01 \mathrm{E}$ & 0.001 & 0.041 & 0.403 & 0.003 & 0.018 & 0.000 & ASA- & 0. \\
\hline 12 & 267 & 079 & 037 & -05 & 417 & 502 & 828 & 778 & 349 & 718 & 12 & 49 \\
\hline aver & 0.029 & 0.001 & 0.029 & $5.23 \mathrm{E}$ & 0.005 & 0.259 & 1.322 & 0.004 & 0.046 & 0.001 & aver & 1. \\
\hline \multirow[t]{2}{*}{ age } & 898 & 529 & 347 & -05 & 698 & 102 & 932 & 927 & 143 & 377 & age & 70 \\
\hline & 0.002 & 0.001 & 0.010 & $1.01 \mathrm{E}$ & 0.001 & 0.031 & 0.039 & 0.002 & 0.018 & 0.000 & & 0. \\
\hline \multirow[t]{2}{*}{$\min$} & 267 & 079 & 814 & -05 & 417 & 41 & 33 & 833 & 349 & 504 & $\min$ & 12 \\
\hline & 0.160 & 0.002 & 0.076 & 0.000 & 0.021 & 0.810 & 5.137 & 0.014 & 0.208 & 0.005 & & 5. \\
\hline $\max$ & 216 & 968 & 691 & 181 & 722 & 603 & 778 & 733 & 317 & 553 & $\max$ & 80 \\
\hline stde & 0.044 & 0.000 & 0.019 & $4.58 \mathrm{E}$ & 0.005 & 0.277 & 1.540 & 0.003 & 0.053 & 0.001 & stde & 1. \\
\hline \multirow[t]{2}{*}{ v } & 767 & 674 & 028 & -05 & 797 & 269 & 605 & 165 & 291 & 403 & v & 74 \\
\hline & 0.002 & 4.55E & 0.000 & 2.1E- & $3.36 \mathrm{E}$ & 0.076 & 2.373 & & 0.002 & 1.97E & & 3. \\
\hline \multirow[t]{2}{*}{ var } & 004 & -07 & 362 & 09 & -05 & 878 & 463 & $1 \mathrm{E}-05$ & 84 & -06 & var & 03 \\
\hline & 0.012 & 0.001 & 0.021 & $3.78 \mathrm{E}$ & 0.003 & 0.123 & 0.767 & 0.004 & 0.024 & 0.000 & & 1. \\
\hline med & 731 & 079 & 281 & -05 & 353 & 857 & 911 & 156 & 286 & 913 & med & 00 \\
\hline
\end{tabular}

Hazard Quotient and Hazard Index for Children: The computed hazard quotient, hazard index together with their descriptive statistical summary are presented in Table 8 and their graphical profile illustrated in Figures 9 and 10 respectively. The average computed values of hazard quotient 
LAUTECH Journal of Civil and Environmental Studies

Volume 6, Issue 1; March 2021

for $\mathrm{Al}, \mathrm{As}, \mathrm{Ba}, \mathrm{Cr}, \mathrm{Cu}, \mathrm{Fe}, \mathrm{Mn}, \mathrm{Ni}, \mathrm{Pb}$ and $\mathrm{Zn}$ are: 0.09497, 0.004857, 0.0932, 0.000166, 0.0181, $0.823,4.202,0.0157,0.147$ and 0.00437 respectively.

Table 8: Computed Hazard Quotient, Hazard Index and Statistical Summary in Children Consumers

\begin{tabular}{|c|c|c|c|c|c|c|c|c|c|c|c|c|}
\hline \multirow{2}{*}{$\begin{array}{l}\text { Samp } \\
\text { le ID }\end{array}$} & \multicolumn{11}{|c|}{ Hazard Quotient (Children) } & \multirow[b]{2}{*}{$\begin{array}{l}\text { Hazard } \\
\text { Index }\end{array}$} \\
\hline & $\mathrm{Al}$ & As & $\mathrm{Ba}$ & $\mathrm{Cr}$ & $\mathrm{Cu}$ & $\mathrm{Fe}$ & $\mathrm{Mn}$ & $\mathrm{Ni}$ & $\mathrm{Pb}$ & $\mathrm{Zn}$ & & \\
\hline & 0.017 & 0.003 & 0.087 & 0.000 & 0.007 & 0.099 & 3.441 & 0.013 & & 0.001 & ASA- & \\
\hline \multirow[t]{2}{*}{ ASA-1 } & 4 & 429 & 558 & 088 & 5 & 771 & 078 & 2 & 0.072 & 92 & 1 & 3.74 \\
\hline & 0.020 & 0.005 & 0.100 & 0.000 & 0.029 & 0.228 & 9.973 & 0.013 & 0.058 & 0.006 & ASA- & \\
\hline \multirow[t]{2}{*}{ ASA-2 } & 76 & 143 & 788 & 096 & 4 & 857 & 67 & 8 & 286 & 6 & 2 & 10.44 \\
\hline & 0.015 & 0.009 & 0.166 & 0.000 & 0.005 & 1.730 & & & 0.161 & 0.001 & ASA- & \\
\hline \multirow[t]{2}{*}{ ASA-3 } & 84 & 429 & 458 & 088 & 4 & 057 & 16.32 & 0.009 & 143 & 6 & 3 & 18.42 \\
\hline & 0.508 & 0.008 & 0.243 & 0.000 & & 2.218 & 7.104 & 0.046 & 0.661 & 0.017 & ASA- & \\
\hline \multirow[t]{2}{*}{ ASA-4 } & 92 & 571 & 606 & 576 & 0.069 & 8 & 522 & 8 & 714 & 64 & 4 & 10.88 \\
\hline & 0.075 & 0.005 & 0.066 & 0.000 & 0.010 & 1.138 & 2.762 & 0.014 & 0.078 & 0.002 & ASA- & \\
\hline \multirow[t]{2}{*}{ ASA-5 } & 96 & 143 & 498 & 144 & 5 & 114 & 452 & 4 & 857 & 12 & 5 & 4.15 \\
\hline & 0.094 & 0.003 & 0.068 & 0.000 & 0.012 & 0.490 & 0.787 & 0.010 & 0.181 & 0.004 & ASA- & \\
\hline \multirow[t]{2}{*}{ ASA- 6} & 2 & 429 & 22 & 104 & 3 & 971 & 174 & 2 & 714 & 44 & 6 & 1.65 \\
\hline & 0.205 & 0.003 & 0.066 & 0.000 & 0.033 & 0.561 & 0.612 & 0.017 & 0.188 & & ASA- & \\
\hline \multirow[t]{2}{*}{ ASA-7 } & 08 & 429 & 978 & 232 & 3 & 086 & 991 & 4 & 571 & 0.005 & 7 & 1.69 \\
\hline & 0.102 & 0.003 & 0.042 & 0.000 & 0.016 & 0.287 & 0.209 & 0.014 & 0.082 & 0.002 & ASA- & \\
\hline \multirow[t]{2}{*}{ ASA- 8} & 6 & 429 & 534 & 264 & 8 & 314 & 687 & 4 & 286 & 04 & 8 & 0.76 \\
\hline & 0.037 & & 0.128 & 0.000 & 0.007 & 2.574 & 5.691 & 0.013 & 0.075 & 0.001 & ASA- & \\
\hline ASA-9 & 68 & 0.006 & 244 & 168 & 5 & 857 & 757 & 2 & 429 & 72 & 9 & 8.54 \\
\hline ASA- & 0.043 & 0.003 & 0.065 & 0.000 & 0.010 & 0.295 & 2.116 & 0.010 & 0.075 & 0.003 & ASA- & \\
\hline 10 & 2 & 429 & 628 & 136 & 2 & 886 & 043 & 8 & 429 & 6 & 10 & 2.62 \\
\hline ASA- & 0.010 & 0.003 & 0.034 & 0.000 & 0.010 & 0.118 & 0.124 & 0.012 & 0.065 & 0.003 & ASA- & \\
\hline 11 & 8 & 429 & 35 & 064 & 8 & 8 & 93 & 6 & 143 & 52 & 11 & 0.38 \\
\hline ASA- & 0.007 & 0.003 & 0.047 & 0.000 & 0.004 & 0.131 & 1.282 & & 0.058 & 0.002 & ASA- & \\
\hline 12 & 2 & 429 & 766 & 032 & 5 & 829 & 748 & 0.012 & 286 & 28 & 12 & 1.55 \\
\hline avera & 0.094 & 0.004 & 0.093 & 0.000 & 0.018 & 0.823 & 4.202 & 0.015 & 0.146 & 0.004 & aver & \\
\hline \multirow[t]{2}{*}{ ge } & 97 & 857 & 219 & 166 & 1 & 029 & 254 & 65 & 571 & 373 & age & 5.40 \\
\hline & 0.007 & 0.003 & 0.034 & 0.000 & 0.004 & 0.099 & 0.124 & & 0.058 & 0.001 & & \\
\hline \multirow[t]{2}{*}{$\min$} & 2 & 429 & 35 & 032 & 5 & 771 & 93 & 0.009 & 286 & 6 & $\min$ & 0.38 \\
\hline & 0.508 & 0.009 & 0.243 & 0.000 & & 2.574 & & 0.046 & 0.661 & 0.017 & & \\
\hline \multirow[t]{2}{*}{$\max$} & 92 & 429 & 606 & 576 & 0.069 & 857 & 16.32 & 8 & 714 & 64 & $\max$ & 18.42 \\
\hline & 0.142 & 0.002 & 0.060 & 0.000 & 0.018 & 0.880 & 4.893 & 0.010 & 0.169 & 0.004 & stde & \\
\hline \multirow[t]{2}{*}{ stdev } & 202 & 142 & 442 & 145 & 413 & 735 & 685 & 054 & 278 & 455 & v & 5.53 \\
\hline & 0.020 & 4.59E & 0.003 & $2.12 \mathrm{E}$ & 0.000 & 0.775 & 23.94 & 0.000 & 0.028 & $1.98 \mathrm{E}$ & & \\
\hline \multirow[t]{2}{*}{ var } & 221 & -06 & 653 & -08 & 339 & 695 & 816 & 101 & 655 & -05 & var & 30.55 \\
\hline & 0.040 & 0.003 & 0.067 & 0.000 & 0.010 & 0.393 & 2.439 & 0.013 & 0.077 & 0.002 & & \\
\hline med & 44 & 429 & 599 & 12 & 65 & 429 & 248 & 2 & 143 & 9 & med & 3.18 \\
\hline
\end{tabular}

Based on the average value, Mn only has hazard quotient value of 4.2 above the recommended value of 1 (USEPA 1989; Su et al., 2017).

However, the individual computed hazard quotient values for Fe in samples ASA-3, ASA-4, ASA5 and ASA-9 are: 1.7, 2.2, 1.1 and 2.6 respectively greater than recommended value of unity. This 
is an indication of health risk to the consumers. The range of the computed hazard index for children in the samples is from 0.38 in sample ASA-11 to 18.4 in sample ASA-3 with an average of 5.4. This shows that the hazard index ranges from low to high health risk to the consumers.

Chronic Daily Intake of Trace Elements in Adults: Table 9 presents the computed chronic daily intake and its statistical summary for Adults consumers.

Table 9: Chronic Daily Intake computed for the Adult Consumers

\begin{tabular}{|c|c|c|c|c|c|c|c|c|c|c|}
\hline \multicolumn{11}{|c|}{ Chronic Daily Intake (CDI) for Adults } \\
\hline $\begin{array}{c}\text { Trace } \\
\text { Elements }\end{array}$ & Al & As & $\mathrm{Ba}$ & $\mathrm{Cr}$ & $\mathrm{Cu}$ & $\mathrm{Fe}$ & $\mathrm{Mn}$ & $\mathrm{Ni}$ & $\mathrm{Pb}$ & $\mathrm{Zn}$ \\
\hline & 0.0056 & $1.55 \mathrm{E}-$ & 0.0056 & $4.27 \mathrm{E}-$ & $9.71 \mathrm{E}-$ & 0.0226 & 0.0512 & $8.55 \mathrm{E}-$ & 8.16E- & 0.0001 \\
\hline \multirow[t]{2}{*}{ ASA-1 } & 34 & 05 & 7 & 05 & 05 & 15 & 56 & 05 & 05 & 87 \\
\hline & 0.0067 & 2.33E- & 0.0065 & 4.66E- & 0.0003 & 0.0518 & 0.1485 & $8.94 \mathrm{E}-$ & 6.61E- & 0.0006 \\
\hline \multirow[t]{2}{*}{ ASA-2 } & 22 & 05 & 27 & 05 & 81 & 74 & 6 & 05 & 05 & 41 \\
\hline & 0.0051 & 4.27E- & 0.0107 & 4.27E- & 6.99E- & 0.3921 & 0.2430 & $5.83 \mathrm{E}-$ & 0.0001 & 0.0001 \\
\hline \multirow[t]{2}{*}{ ASA-3 } & 29 & 05 & 8 & 05 & 05 & 46 & 9 & 05 & 83 & 55 \\
\hline & 0.1647 & 3.89E- & 0.0157 & 0.0002 & 0.0008 & 0.5029 & 0.1058 & 0.0003 & 0.0007 & 0.0017 \\
\hline \multirow[t]{2}{*}{ ASA-4 } & 93 & 05 & 76 & 8 & 94 & 28 & 24 & 03 & 5 & 14 \\
\hline & 0.0245 & $2.33 \mathrm{E}-$ & 0.0043 & $6.99 \mathrm{E}-$ & 0.0001 & 0.2579 & 0.0411 & $9.33 \mathrm{E}-$ & $8.94 \mathrm{E}-$ & 0.0002 \\
\hline \multirow[t]{2}{*}{ ASA-5 } & 97 & 05 & 07 & 05 & 36 & 73 & 47 & 05 & 05 & 06 \\
\hline & 0.0305 & $1.55 \mathrm{E}-$ & 0.0044 & 5.05E- & 0.0001 & 0.1112 & 0.0117 & $6.61 \mathrm{E}-$ & 0.0002 & 0.0004 \\
\hline \multirow[t]{2}{*}{ ASA-6 } & 03 & 05 & 18 & 05 & 59 & 87 & 25 & 05 & 06 & 31 \\
\hline & 0.0664 & $1.55 \mathrm{E}-$ & 0.0043 & 0.0001 & 0.0004 & 0.1271 & 0.0091 & 0.0001 & 0.0002 & 0.0004 \\
\hline \multirow[t]{2}{*}{ ASA-7 } & 07 & 05 & 38 & 13 & 31 & 79 & 31 & 13 & 14 & 86 \\
\hline & 0.0332 & $1.55 \mathrm{E}-$ & 0.0027 & 0.0001 & 0.0002 & 0.0651 & 0.0031 & $9.33 \mathrm{E}-$ & 9.33E- & 0.0001 \\
\hline \multirow[t]{2}{*}{ ASA-8 } & 23 & 05 & 55 & 28 & 18 & 25 & 23 & 05 & 05 & 98 \\
\hline & 0.0122 & $2.72 \mathrm{E}-$ & 0.0083 & 8.16E- & $9.71 \mathrm{E}-$ & 0.5836 & 0.0847 & $8.55 \mathrm{E}-$ & 8.55E- & 0.0001 \\
\hline \multirow[t]{2}{*}{ ASA-9 } & 01 & 05 & 05 & 05 & 05 & 34 & 8 & 05 & 05 & 67 \\
\hline & 0.0139 & $1.55 \mathrm{E}-$ & 0.0042 & $6.61 \mathrm{E}-$ & 0.0001 & 0.0670 & 0.0315 & $6.99 \mathrm{E}-$ & $8.55 \mathrm{E}-$ & 0.0003 \\
\hline \multirow[t]{2}{*}{ ASA-10 } & 89 & 05 & 5 & 05 & 32 & 67 & 19 & 05 & 05 & 5 \\
\hline & 0.0034 & $1.55 \mathrm{E}-$ & 0.0022 & $3.11 \mathrm{E}-$ & 0.0001 & 0.0269 & 0.0018 & $8.16 \mathrm{E}-$ & 7.38E- & 0.0003 \\
\hline \multirow[t]{2}{*}{ ASA-11 } & 97 & 05 & 25 & 05 & 4 & 28 & 61 & 05 & 05 & 42 \\
\hline & 0.0023 & $1.55 \mathrm{E}-$ & 0.0030 & $1.55 \mathrm{E}-$ & 5.83E- & 0.0298 & 0.0191 & 7.77E- & 6.61E- & 0.0002 \\
\hline \multirow[t]{2}{*}{ ASA-12 } & 31 & 05 & 93 & 05 & 05 & 81 & 07 & 05 & 05 & 21 \\
\hline & 0.0307 & $2.2 \mathrm{E}-$ & 0.0060 & 8.06E- & 0.0002 & 0.1865 & 0.0625 & 0.0001 & 0.0001 & 0.0004 \\
\hline \multirow[t]{2}{*}{ average } & 52 & 05 & 37 & 05 & 34 & 53 & 94 & 01 & 66 & 25 \\
\hline & 0.0023 & $1.55 \mathrm{E}-$ & 0.0022 & $1.55 \mathrm{E}-$ & $5.83 \mathrm{E}-$ & 0.0226 & 0.0018 & $5.83 \mathrm{E}-$ & $6.61 \mathrm{E}-$ & 0.0001 \\
\hline \multirow[t]{2}{*}{$\min$} & 31 & 05 & 25 & 05 & 05 & 15 & 61 & 05 & 05 & 55 \\
\hline & 0.1647 & 4.27E- & 0.0157 & 0.0002 & 0.0008 & 0.5836 & 0.2430 & 0.0003 & 0.0007 & 0.0017 \\
\hline \multirow[t]{2}{*}{$\max$} & 93 & 05 & 76 & 8 & 94 & 34 & 9 & 03 & 5 & 14 \\
\hline & 0.0460 & $9.71 \mathrm{E}-$ & 0.0039 & 7.06E- & 0.0002 & 0.1996 & 0.0728 & $6.51 \mathrm{E}-$ & 0.0001 & 0.0004 \\
\hline \multirow[t]{2}{*}{ stdev } & 46 & 06 & 14 & 05 & 38 & 33 & 93 & 05 & 92 & 33 \\
\hline & 0.0021 & $9.43 \mathrm{E}-$ & $1.53 \mathrm{E}-$ & 4.99E- & 5.69E- & 0.0398 & 0.0053 & $4.24 \mathrm{E}-$ & $3.68 \mathrm{E}-$ & $1.87 \mathrm{E}-$ \\
\hline \multirow[t]{2}{*}{ var } & 2 & 11 & 05 & 09 & 08 & 53 & 13 & 09 & 08 & 07 \\
\hline & 0.0130 & $1.55 \mathrm{E}-$ & 0.0043 & 5.83E- & 0.0001 & 0.0891 & 0.0363 & $8.55 \mathrm{E}-$ & $8.74 \mathrm{E}-$ & 0.0002 \\
\hline med & 95 & 05 & 78 & 05 & 38 & 77 & 33 & 05 & 05 & 82 \\
\hline
\end{tabular}


LAUTECH Journal of Civil and Environmental Studies

Volume 6, Issue 1; March 2021

The average values computed for $\mathrm{Al}, \mathrm{As}, \mathrm{Ba}, \mathrm{Cr}, \mathrm{Cu}, \mathrm{Fe}, \mathrm{Mn}, \mathrm{Ni}, \mathrm{Pb}$ and $\mathrm{Zn}$ are: 0.03, 2.2E-05, 0.006, 8.06E-05, 0.000234, 0.187, 0.063, 0.000101, 0.000166 and 0.000425 respectively.

Values computed for Fe are generally higher than other elements. In general, the average values of the trace elements are less than unity below the recommended value. Figures 11 and 12 illustrate the profiles of chronic daily intakes in Adults.

Chronic Daily Intake of Trace Elements in Children: The computed chronic daily intake for children is presented in Table 10 and the graphical profiles are illustrated in Figures 13 and 14.

Table 10: Chronic Daily Intake computed for the Children

\begin{tabular}{|c|c|c|c|c|c|c|c|c|c|c|}
\hline \multicolumn{11}{|c|}{ Chronic Daily Intake (CDI) for Children } \\
\hline Sample & & & & & & & & & & \\
\hline ID & Al & As & $\mathrm{Ba}$ & $\mathrm{Cr}$ & $\mathrm{Cu}$ & Fe & Mn & $\mathrm{Ni}$ & $\mathrm{Pb}$ & Zn \\
\hline & & 0.0000 & 0.0175 & 0.0001 & & 0.0698 & 0.1582 & 0.0002 & 0.0002 & 0.0005 \\
\hline \multirow[t]{2}{*}{ ASA-1 } & 0.0174 & 48 & 12 & 32 & 0.0003 & 4 & 9 & 64 & 52 & 76 \\
\hline & 0.0207 & 0.0000 & 0.0201 & 0.0001 & 0.0011 & & 0.4587 & 0.0002 & 0.0002 & 0.0019 \\
\hline \multirow[t]{2}{*}{ ASA-2 } & 6 & 72 & 58 & 44 & 76 & 0.1602 & 89 & 76 & 04 & 8 \\
\hline & 0.0158 & 0.0001 & 0.0332 & 0.0001 & 0.0002 & 1.2110 & 0.7507 & 0.0001 & 0.0005 & 0.0004 \\
\hline \multirow[t]{2}{*}{ ASA-3 } & 4 & 32 & 92 & 32 & 16 & 4 & 2 & 8 & 64 & 8 \\
\hline & 0.5089 & 0.0001 & 0.0487 & 0.0008 & 0.0027 & 1.5531 & 0.3268 & 0.0009 & 0.0023 & 0.0052 \\
\hline \multirow[t]{2}{*}{ ASA-4 } & 2 & 2 & 21 & 64 & 6 & 6 & 08 & 36 & 16 & 92 \\
\hline & 0.0759 & 0.0000 & & 0.0002 & 0.0004 & 0.7966 & 0.1270 & 0.0002 & 0.0002 & 0.0006 \\
\hline \multirow[t]{2}{*}{ ASA-5 } & 6 & 72 & 0.0133 & 16 & 2 & 8 & 73 & 88 & 76 & 36 \\
\hline & & 0.0000 & 0.0136 & 0.0001 & 0.0004 & 0.3436 & 0.0362 & 0.0002 & 0.0006 & 0.0013 \\
\hline \multirow[t]{2}{*}{ ASA-6 } & 0.0942 & 48 & 44 & 56 & 92 & 8 & 1 & 04 & 36 & 32 \\
\hline & 0.2050 & 0.0000 & 0.0133 & 0.0003 & 0.0013 & 0.3927 & 0.0281 & 0.0003 & 0.0006 & \\
\hline \multirow[t]{2}{*}{ ASA-7 } & 8 & 48 & 96 & 48 & 32 & 6 & 98 & 48 & 6 & 0.0015 \\
\hline & & 0.0000 & 0.0085 & 0.0003 & 0.0006 & 0.2011 & 0.0096 & 0.0002 & 0.0002 & 0.0006 \\
\hline \multirow[t]{2}{*}{ ASA-8 } & 0.1026 & 48 & 07 & 96 & 72 & 2 & 46 & 88 & 88 & 12 \\
\hline & 0.0376 & 0.0000 & 0.0256 & 0.0002 & & & 0.2618 & 0.0002 & 0.0002 & 0.0005 \\
\hline \multirow[t]{2}{*}{ ASA-9 } & 8 & 84 & 49 & 52 & 0.0003 & 1.8024 & 21 & 64 & 64 & 16 \\
\hline & & 0.0000 & 0.0131 & 0.0002 & 0.0004 & 0.2071 & 0.0973 & 0.0002 & 0.0002 & 0.0010 \\
\hline \multirow[t]{2}{*}{ ASA-10 } & 0.0432 & 48 & 26 & 04 & 08 & 2 & 38 & 16 & 64 & 8 \\
\hline & & 0.0000 & 0.0068 & 0.0000 & 0.0004 & 0.0831 & 0.0057 & 0.0002 & 0.0002 & 0.0010 \\
\hline \multirow[t]{2}{*}{ ASA-11 } & 0.0108 & 48 & 7 & 96 & 32 & 6 & 47 & 52 & 28 & 56 \\
\hline & & 0.0000 & 0.0095 & 0.0000 & 0.0001 & 0.0922 & 0.0590 & 0.0002 & 0.0002 & 0.0006 \\
\hline ASA-12 & 0.0072 & 48 & 53 & 48 & 8 & 8 & 06 & 4 & 04 & 84 \\
\hline averag & 0.0949 & 0.0000 & 0.0186 & 0.0002 & 0.0007 & 0.5761 & 0.1933 & 0.0003 & 0.0005 & 0.0013 \\
\hline \multirow[t]{2}{*}{ e } & 7 & 68 & 44 & 49 & 24 & 2 & 04 & 13 & 13 & 12 \\
\hline & & 0.0000 & 0.0068 & 0.0000 & 0.0001 & 0.0698 & 0.0057 & 0.0001 & 0.0002 & 0.0004 \\
\hline \multirow[t]{2}{*}{$\min$} & 0.0072 & 48 & 7 & 48 & 8 & 4 & 47 & 8 & 04 & 8 \\
\hline & 0.5089 & 0.0001 & 0.0487 & 0.0008 & 0.0027 & & 0.7507 & 0.0009 & 0.0023 & 0.0052 \\
\hline \multirow[t]{2}{*}{$\max$} & 2 & 32 & 21 & 64 & 6 & 1.8024 & 2 & 36 & 16 & 92 \\
\hline & 0.1422 & & 0.0120 & 0.0002 & 0.0007 & 0.6165 & 0.2251 & 0.0002 & 0.0005 & 0.0013 \\
\hline \multirow[t]{2}{*}{ stdev } & 02 & $3 \mathrm{E}-05$ & 88 & 18 & 37 & 15 & 1 & 01 & 92 & 37 \\
\hline & 0.0202 & 8.99E- & 0.0001 & 4.76E- & $5.42 \mathrm{E}-$ & 0.3800 & 0.0506 & 4.04E- & 3.51E- & $1.79 \mathrm{E}-$ \\
\hline \multirow[t]{2}{*}{ var } & 21 & 10 & 46 & 08 & 07 & 9 & 74 & 08 & 07 & 06 \\
\hline & 0.0404 & 0.0000 & 0.0135 & 0.0001 & 0.0004 & & 0.1122 & 0.0002 & 0.0002 & 0.0008 \\
\hline med & 4 & 48 & 2 & 8 & 26 & 0.2754 & 05 & 64 & 7 & 7 \\
\hline
\end{tabular}


The following are the average values for $\mathrm{Al}, \mathrm{As}, \mathrm{Ba}, \mathrm{Cr}, \mathrm{Cu}, \mathrm{Fe}, \mathrm{Mn}, \mathrm{Ni}, \mathrm{Pb}$ and $\mathrm{Zn}$ respectively: $0.095,0.000068,0.017,0.00025,0.00072,0.58,0.19,0.00031,0.00051$ and 0.0013 . Based on the average values, they are lower than the recommended values of unity.

However, individual values computed for Fe have chronic daily intake higher than unity above the recommended values in samples: ASA-3 (1.2), ASA-4 (1.6) and ASA-9 (1.8). As illustrated by Figure 13, samples ASA-3, ASA-4 and ASA-9 are more loaded with the trace elements, while Fe generally takes the lead in the three samples. Hence, care must be taken for children consuming the water in the area.

Carcinogenic Risk for Adults and Children: Two carcinogenic elements are considered in this research namely $\mathrm{Cr}$ and $\mathrm{Pb}$. The computed values are presented in Table 11 together with their statistical summary and their graphical profiles are also presented in Figures 15 and 16. In adults, $\mathrm{Cr}$ ranges from 0.00003 to 0.0005 with an average of 0.0002 while $\mathrm{Pb}$ ranges from 0.008 to 0.09 with an average of 0.02 . From the values computed for children, $\mathrm{Cr}$ ranges from 0.0001 to 0.002 with an average of 0.00005 while $\mathrm{Pb}$ ranges from 0.02 to 0.3 with an average of 0.06 . However, the average carcinogenic risk values exceeded the prescribed limit of $10^{-6}$ and $10^{-4}$ respectively (Edokpayi et al., 2018). This is likely to pose potential carcinogenic health risk hazard to both adults and children consuming the water in the investigated area. Hence, adequate precaution needs to be taken to avoid potential carcinogenic risk of people in the area especially, children consuming the water resources.

Table 11: Computed Carcinogenic Risk values and their Descriptive Statistical Summary in Adults and Children

\begin{tabular}{lllll}
\hline \multicolumn{2}{c}{ Sample ID } & \multicolumn{2}{c}{ Carcinogenic risk (Adult) } & \multicolumn{2}{c}{ Carcinogenic risk (Children) } \\
& $\mathrm{Cr}$ & $\mathrm{Pb}$ & $\mathrm{Cr}$ & $\mathrm{Pb}$ \\
\hline \hline ASA-1 & $8 \mathrm{E}-05$ & $9 \mathrm{E}-03$ & $3 \mathrm{E}-04$ & $3 \mathrm{E}-02$ \\
$\mathrm{ASA}-2$ & $9 \mathrm{E}-05$ & $8 \mathrm{E}-03$ & $3 \mathrm{E}-04$ & $2 \mathrm{E}-02$ \\
ASA-3 & $8 \mathrm{E}-05$ & $2 \mathrm{E}-02$ & $3 \mathrm{E}-04$ & $7 \mathrm{E}-02$ \\
ASA-4 & $5 \mathrm{E}-04$ & $9 \mathrm{E}-02$ & $2 \mathrm{E}-03$ & $3 \mathrm{E}-01$ \\
ASA-5 & $1 \mathrm{E}-04$ & $1 \mathrm{E}-02$ & $4 \mathrm{E}-04$ & $3 \mathrm{E}-02$ \\
ASA-6 & $1 \mathrm{E}-04$ & $2 \mathrm{E}-02$ & $3 \mathrm{E}-04$ & $7 \mathrm{E}-02$ \\
ASA-7 & $2 \mathrm{E}-04$ & $2 \mathrm{E}-02$ & $7 \mathrm{E}-04$ & $8 \mathrm{E}-02$ \\
ASA-8 & $2 \mathrm{E}-04$ & $1 \mathrm{E}-02$ & $8 \mathrm{E}-04$ & $3 \mathrm{E}-02$ \\
ASA-9 & $2 \mathrm{E}-04$ & $1 \mathrm{E}-02$ & $5 \mathrm{E}-04$ & $3 \mathrm{E}-02$ \\
ASA-10 & $1 \mathrm{E}-04$ & $1 \mathrm{E}-02$ & $4 \mathrm{E}-04$ & $3 \mathrm{E}-02$ \\
ASA-11 & $6 \mathrm{E}-05$ & $8 \mathrm{E}-03$ & $2 \mathrm{E}-04$ & $3 \mathrm{E}-02$ \\
ASA-12 & $3 \mathrm{E}-05$ & $8 \mathrm{E}-03$ & $1 \mathrm{E}-04$ & $2 \mathrm{E}-02$ \\
\hline average & $2 \mathrm{E}-04$ & $2 \mathrm{E}-02$ & $5 \mathrm{E}-04$ & $6 \mathrm{E}-02$ \\
min & $3 \mathrm{E}-05$ & $8 \mathrm{E}-03$ & $1 \mathrm{E}-04$ & $2 \mathrm{E}-02$ \\
max & $5 \mathrm{E}-04$ & $9 \mathrm{E}-02$ & $2 \mathrm{E}-03$ & $3 \mathrm{E}-01$ \\
stdev & $1 \mathrm{E}-04$ & $2 \mathrm{E}-02$ & $4 \mathrm{E}-04$ & $7 \mathrm{E}-02$ \\
var & $2 \mathrm{E}-08$ & $5 \mathrm{E}-04$ & $2 \mathrm{E}-07$ & $5 \mathrm{E}-03$ \\
med & $1 \mathrm{E}-04$ & $1 \mathrm{E}-02$ & $4 \mathrm{E}-04$ & $3 \mathrm{E}-02$ \\
\hline \hline
\end{tabular}

Correlation Matrix: The correlation matrix between elements in water samples establish the interrelationship between the elements and this can be used to predict the source of the elements in the sampled water. Table 12 presents the correlation matrix of the selected trace elements in the 
LAUTECH Journal of Civil and Environmental Studies

Volume 6, Issue 1; March 2021

water samples. Al has positive correlations with all the other trace elements ranging from 0.01 in $\mathrm{Mn}$ to 0.96 in $\mathrm{Cr}$ and this is an indication of interrelationship. Fe has positive correlations with all the other trace elements ranging from 0.32 in $\mathrm{Cu}$ to as high as 0.80 in As. Moreover, Mn has positive correlations with all other trace elements ranging from 0.01 in Al to 0.86 in As. The results of the correlation matrix support the findings from the computed contamination factor of some of the selected trace elements, that the elements are sourced from both weathering of surrounding rock types and various anthropogenic activities in the area of study.

Table 12: Correlation Matrix of the Selected Trace Elements in the Analyzed Water Samples of ASA River Water

\begin{tabular}{|c|c|c|c|c|c|c|c|c|c|c|}
\hline $\begin{array}{c}\text { parameters } \\
(\mathrm{ppb})\end{array}$ & $\mathrm{Al}$ & As & $\mathrm{Ba}$ & $\mathrm{Cr}$ & $\mathrm{Cu}$ & $\mathrm{Fe}$ & $\mathrm{Mn}$ & $\mathrm{Ni}$ & $\mathrm{Pb}$ & $\mathrm{Zn}$ \\
\hline $\mathrm{Al}$ & 1.00 & & & & & & & & & \\
\hline As & 0.40 & 1.00 & & & & & & & & \\
\hline $\mathrm{Ba}$ & 0.66 & 0.89 & 1.00 & & & & & & & \\
\hline $\mathrm{Cr}$ & 0.96 & 0.43 & 0.66 & 1.00 & & & & & & \\
\hline $\mathrm{Cu}$ & 0.93 & 0.38 & 0.64 & 0.89 & 1.00 & & & & & \\
\hline $\mathrm{Fe}$ & 0.45 & 0.80 & 0.78 & 0.52 & 0.32 & 1.00 & & & & \\
\hline $\mathrm{Mn}$ & 0.01 & 0.86 & 0.70 & 0.05 & 0.12 & 0.52 & 1.00 & & & \\
\hline $\mathrm{Ni}$ & 0.95 & 0.46 & 0.71 & 0.93 & 0.92 & 0.46 & 0.09 & 1.00 & & \\
\hline $\mathrm{Pb}$ & 0.95 & 0.57 & 0.80 & 0.89 & 0.87 & 0.52 & 0.21 & 0.93 & 1.00 & \\
\hline $\mathrm{Zn}$ & 0.90 & 0.43 & 0.69 & 0.83 & 0.95 & 0.34 & 0.15 & 0.93 & 0.92 & 1.00 \\
\hline
\end{tabular}

\section{Conclusion and Recommendations}

The preliminary health risk assessment of Asa river water has been established in this research using ten selected trace elements from the hydrochemical data analyzed from the water samples in the area of study. The following conclusions were drawn:

i. The average concentrations of $\mathrm{Al}(0.79 \mathrm{ppm}), \mathrm{Fe}$ (4.8) and $\mathrm{Mn}(1.6)$ show elevated values above the prescribed values of WHO (2006) and NSDWQ (2007), other trace elements are less than the prescribed values.

ii. The computed contamination factors of $\mathrm{Al}(0.2$ - 13.3), $\mathrm{Fe}(8.8$ - 227.6) and $\mathrm{Mn}$ (114 14895.2) ranged from low contamination factor to very high contamination factor and the degree of contamination is extremely high (average $=3910.6)$ ).

iii. The exposure dose through ingestion in adults and children has high average values greater than unity in Al (adults $=29.9 \mu \mathrm{g} / \mathrm{kg} / \mathrm{day}$, children $=95 \mu \mathrm{g} / \mathrm{kg} / \mathrm{day}$ ), $\mathrm{Ba}$ (adults $=5.9$ $\mu \mathrm{g} / \mathrm{kg} / \mathrm{day}$, children $=18.6 \mu \mathrm{g} / \mathrm{kg} / \mathrm{day}), \mathrm{Fe}$ (adults $=181 \mu \mathrm{g} / \mathrm{kg} /$ day, children $=576 \mu \mathrm{g} / \mathrm{kg} / \mathrm{day}$ ) and $\mathrm{Mn}$ (adults $=60.9 \mu \mathrm{g} / \mathrm{kg} / \mathrm{day}$, children $=193 \mu \mathrm{g} / \mathrm{kg} / \mathrm{day}$ ) while other selected trace elements have values below unity except $\mathrm{Zn}(1.3 \mu \mathrm{g} / \mathrm{kg} / \mathrm{day})$ in children that has values greater than unity.

iv. The computed hazard quotient in $\mathrm{Mn}$ is higher than unity while others are less than unity in both adults and children.

v. The average values of hazard index for both age groups are greater than unity, above the prescribed standard of USEPA, which makes the water practically non-carcinogenic health risky to the consumers. 
vi. The average chronic daily intake computed are generally less than unity. However, in children, the computed values for Fe in some samples are higher than unity making the water of potential health risk for the consumers, especially the children.

vii. The average carcinogenic risk values computed for $\mathrm{Cr}$ and $\mathrm{Pb}$ exceeded the recommended values of $10^{-6}$ and $10^{-4}$ respectively. This could pose serious health hazard to the consumers most especially the children having low immunity.

viii. The results of the correlation matrix and contamination factors show that the sources of the trace elements in the river is mainly from both weathering of rock types and anthropogenic activities in the area of study.

Hence, it is recommended that the use of agrochemical by farmers and indiscriminate channeling and dumping of wastes (industrial, domestic and agricultural) into the river should be monitored and regulated.

\section{Acknowledgement}

The authors wish to appreciate the following people for their assistance during the field and laboratory activities: Azeez Rahmat Ayobami, Adelani Akeem Adeola, Kamorudeen Saheedat Adeyemo, Okebaram Uchechukwu David, Kolo Lami Gladys, Adeniyi Adebimpe Comfort, Adeniran Habibullah Ajibola, Ayanwande Philip Ayanlade and Oladele Oluwayomi.

\section{Appendix}

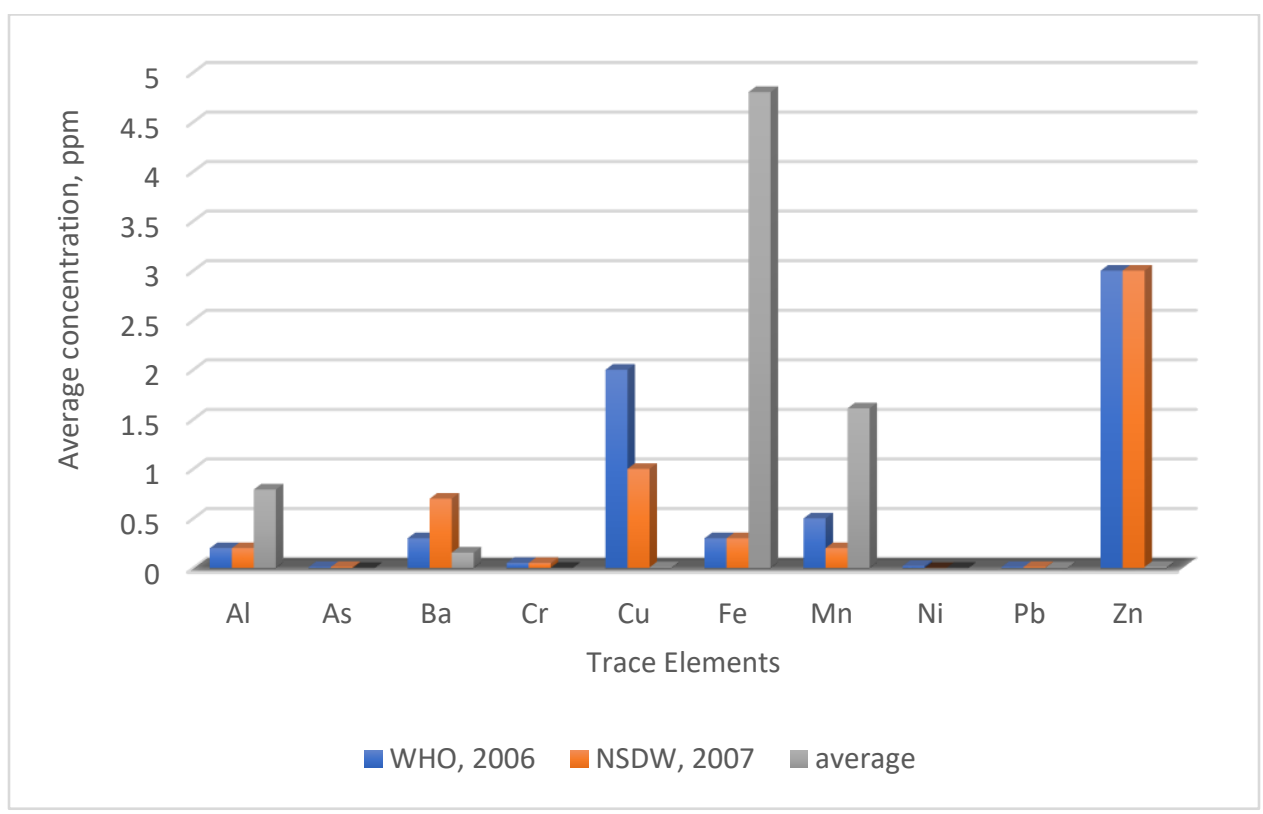

Figure 2: Profile of the Average Concentrations of the Selected Trace Elements Compared with WHO (2006) and NSDWQ (2007) 


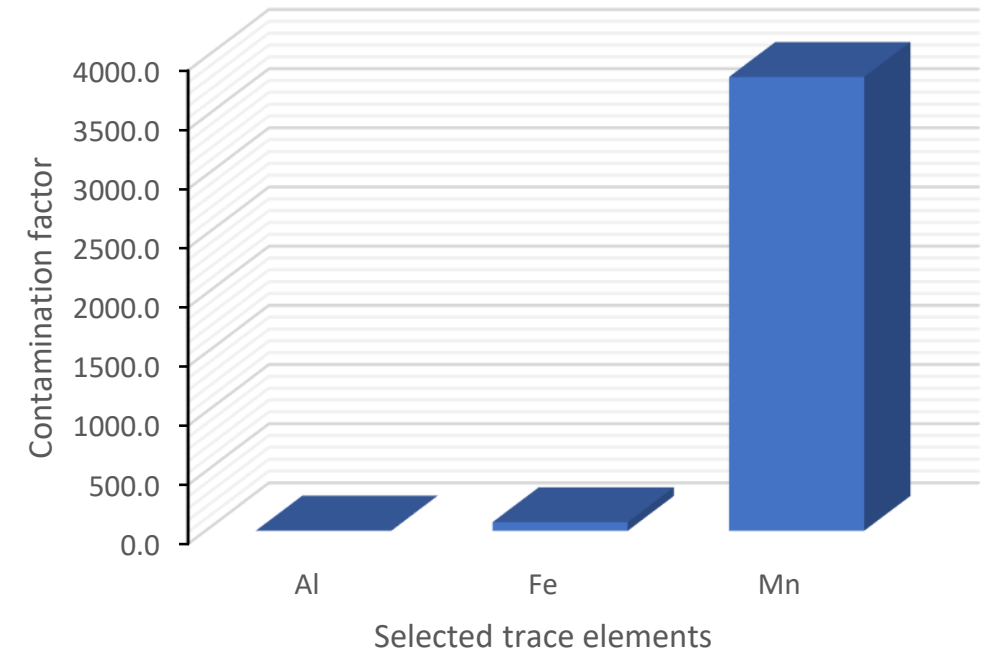

average

Figure 3: Profile of Average Contamination Factor of $\mathrm{Al}, \mathrm{Fe}$ and $\mathrm{Mn}$

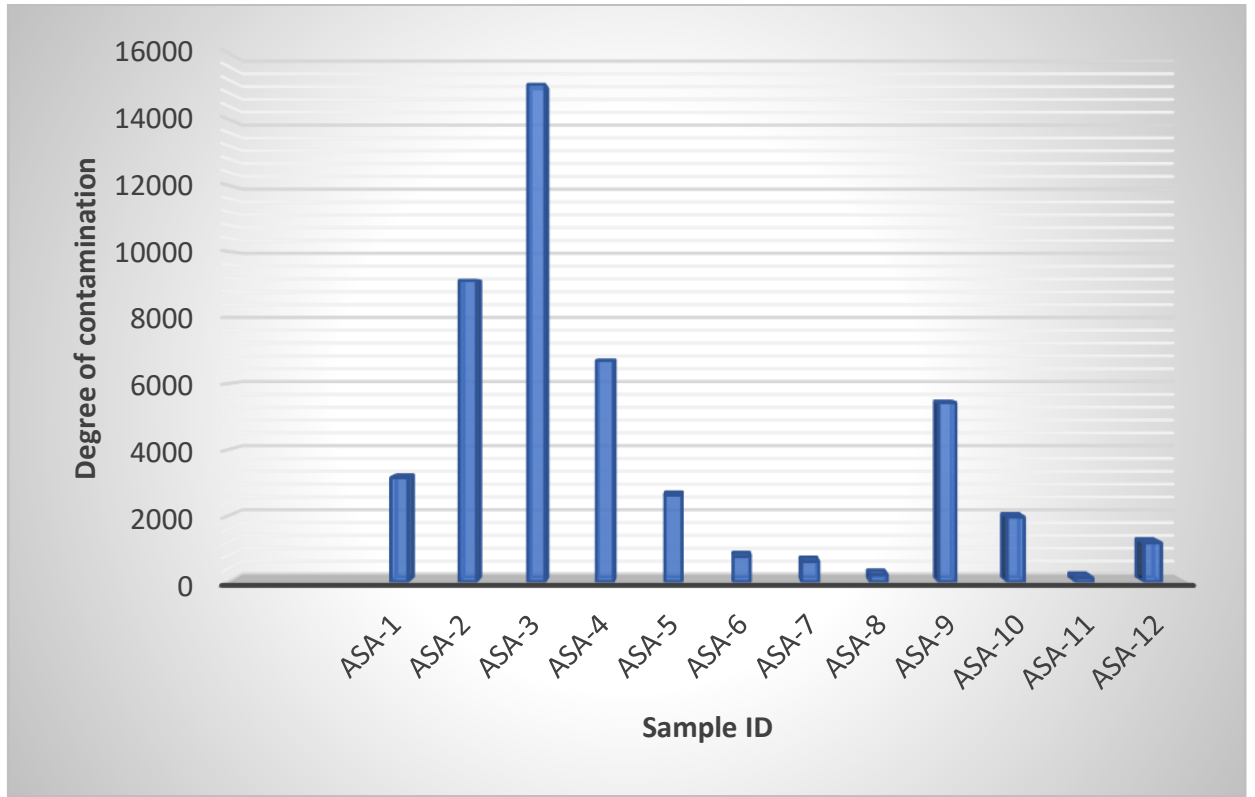

Figure 4: Profile of Degree of Contamination of $\mathrm{Al}, \mathrm{Fe}$ and $\mathrm{Mn}$ 


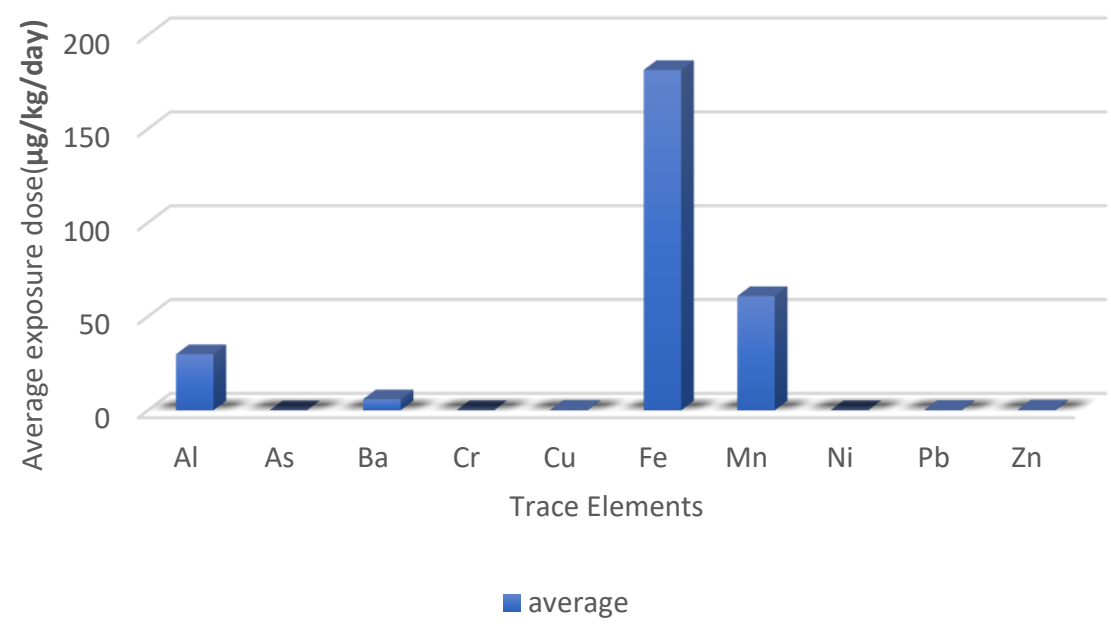

Figure 5: Profile of Exposure Dose through Ingestion in Adults

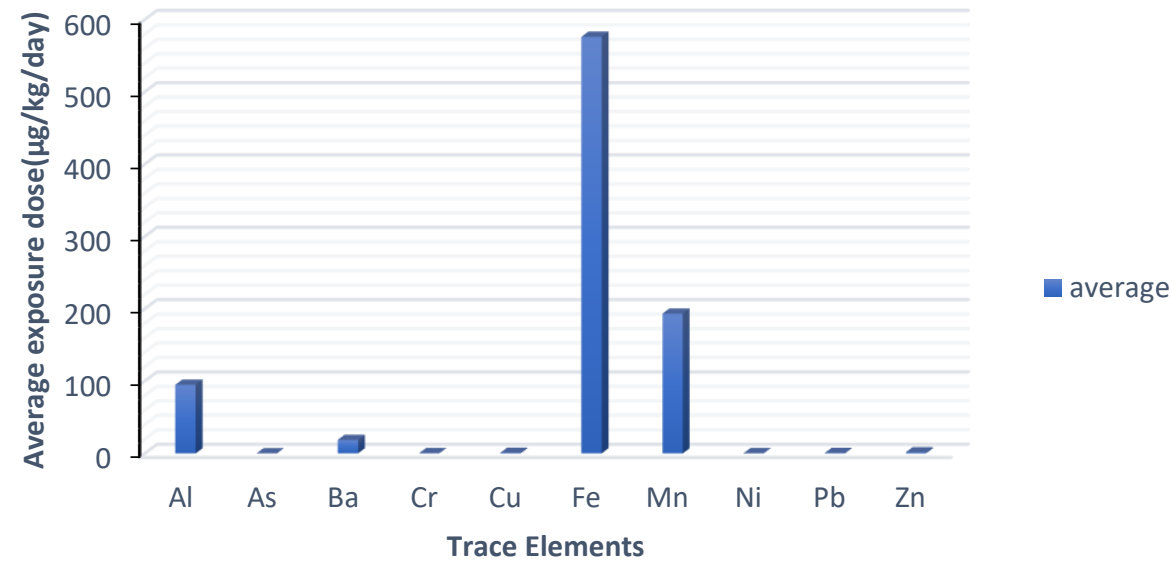

Figure 6: Profile of Exposure Dose through Ingestion in Children

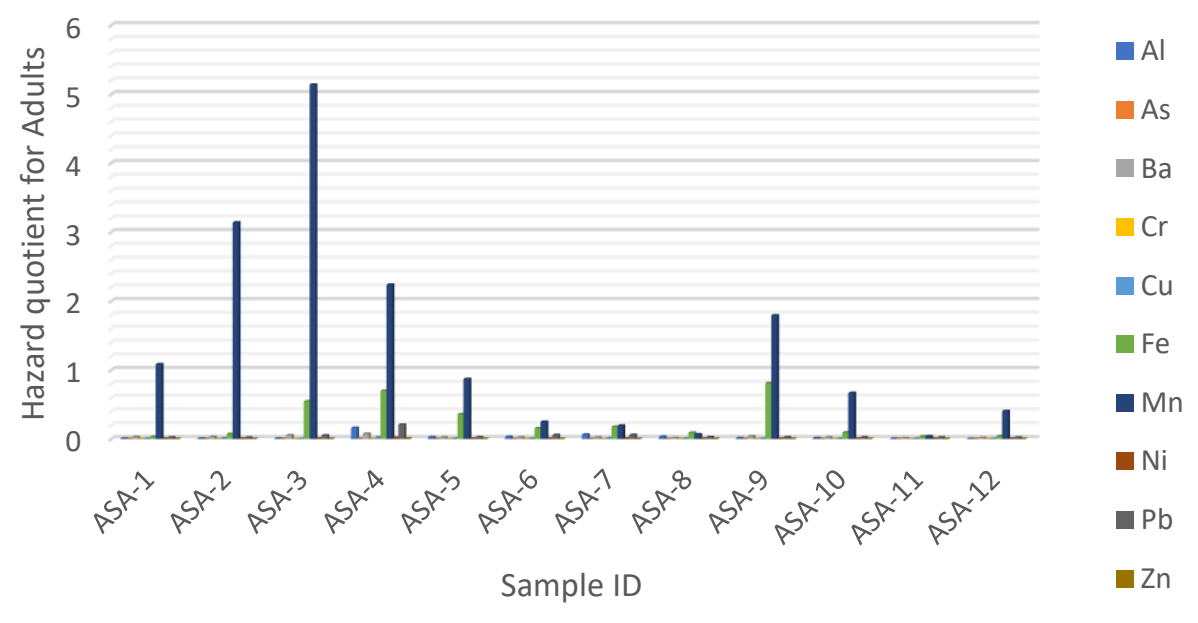

Figure 7: Profile of Hazard Quotient in the Water Samples for Adults 


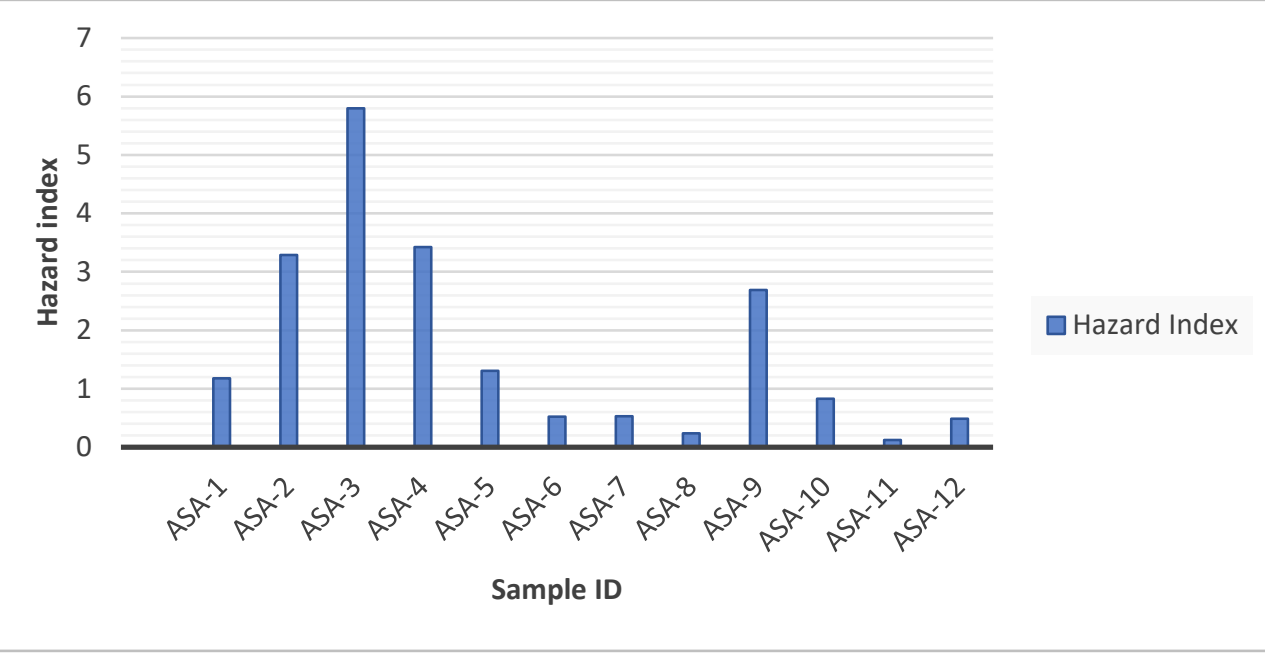

Figure 8: Hazard Index of the Samples Water in Adults

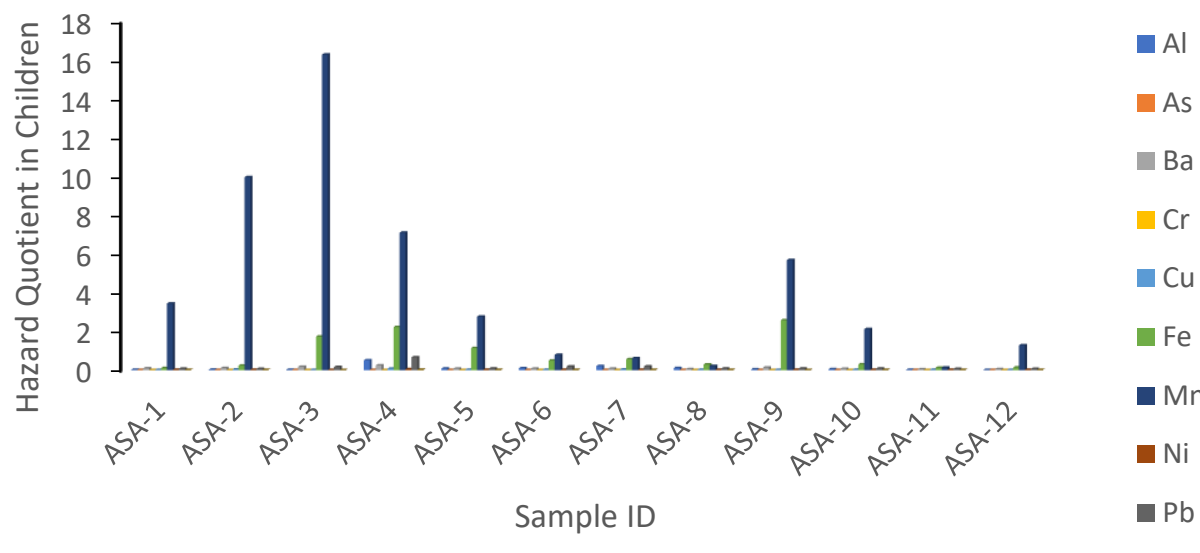

Figure 9: Profile of Hazard Quotient for Children

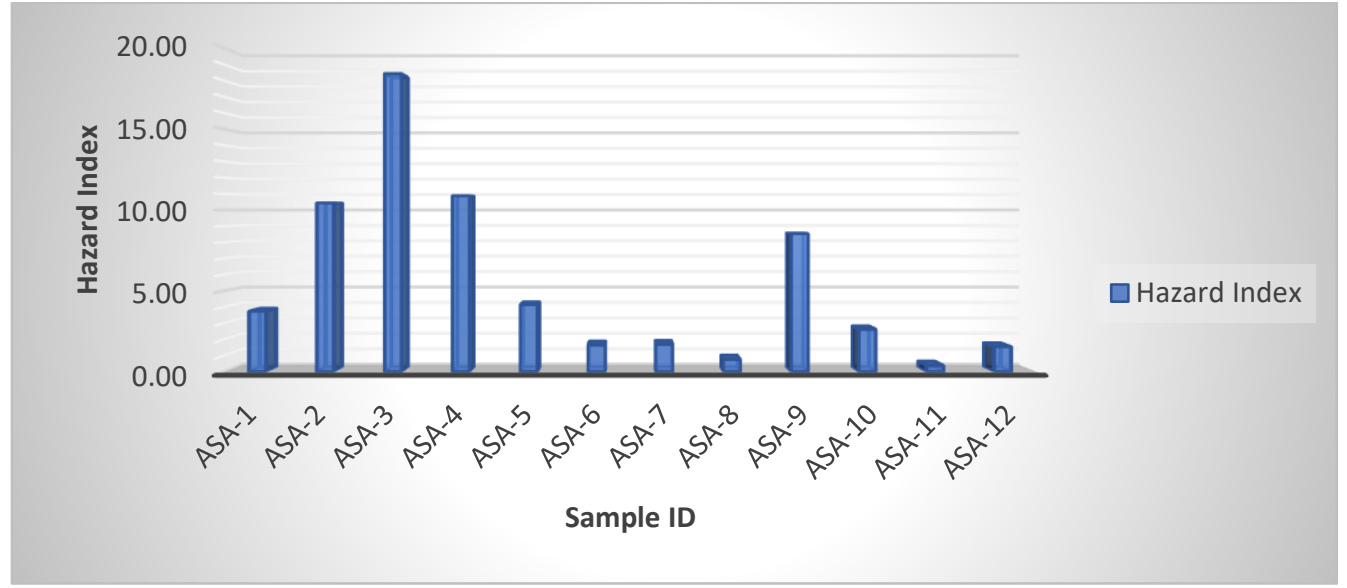

Figure 10: Profile of Hazard Index of the Water Samples for Children 


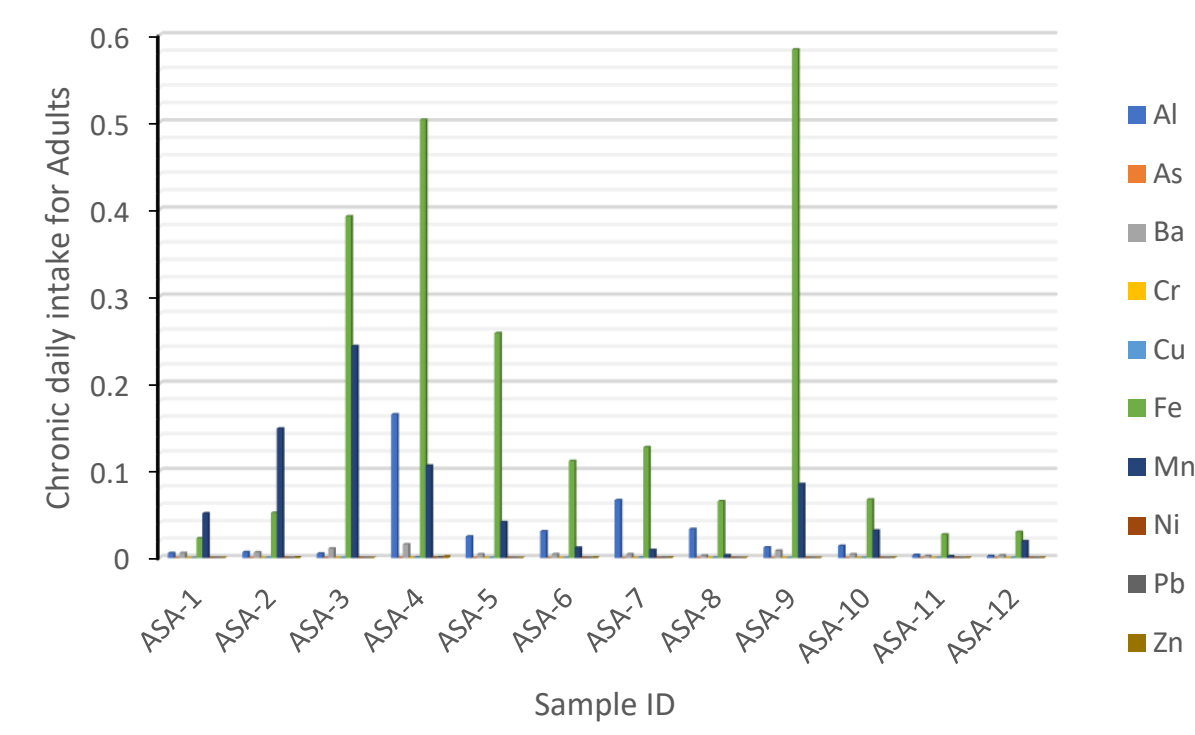

Figure 11: Profile of Chronic Daily Intake for Adults

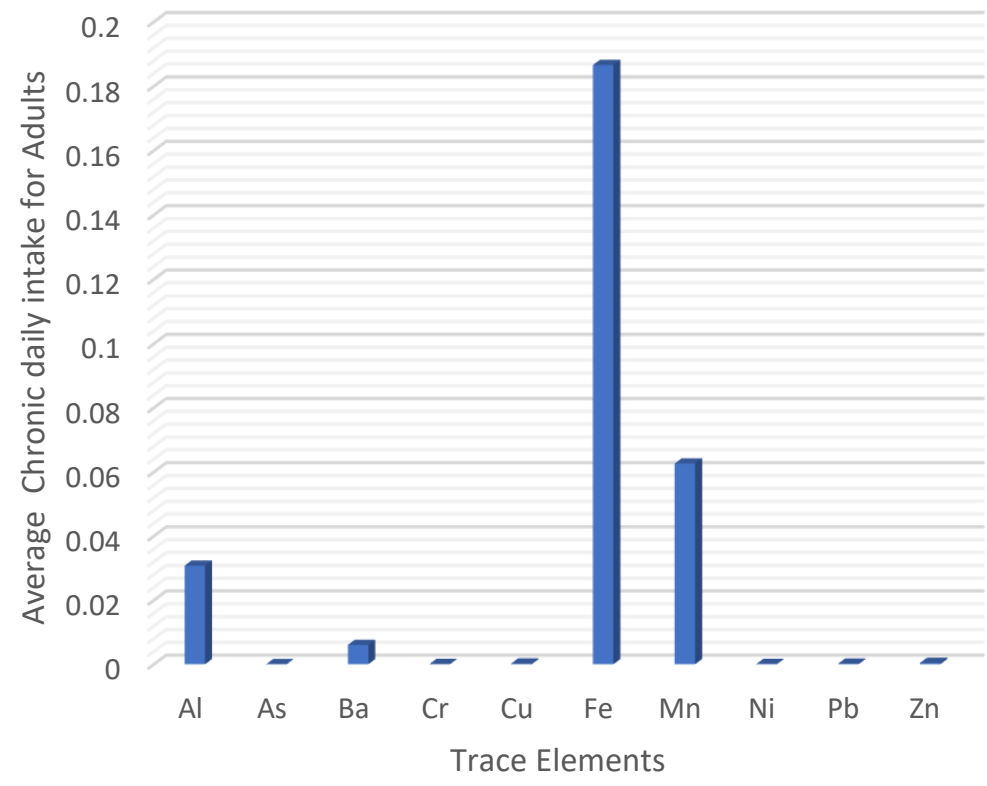

average

Figure 12: Profile of Average Chronic Intake for Adults 


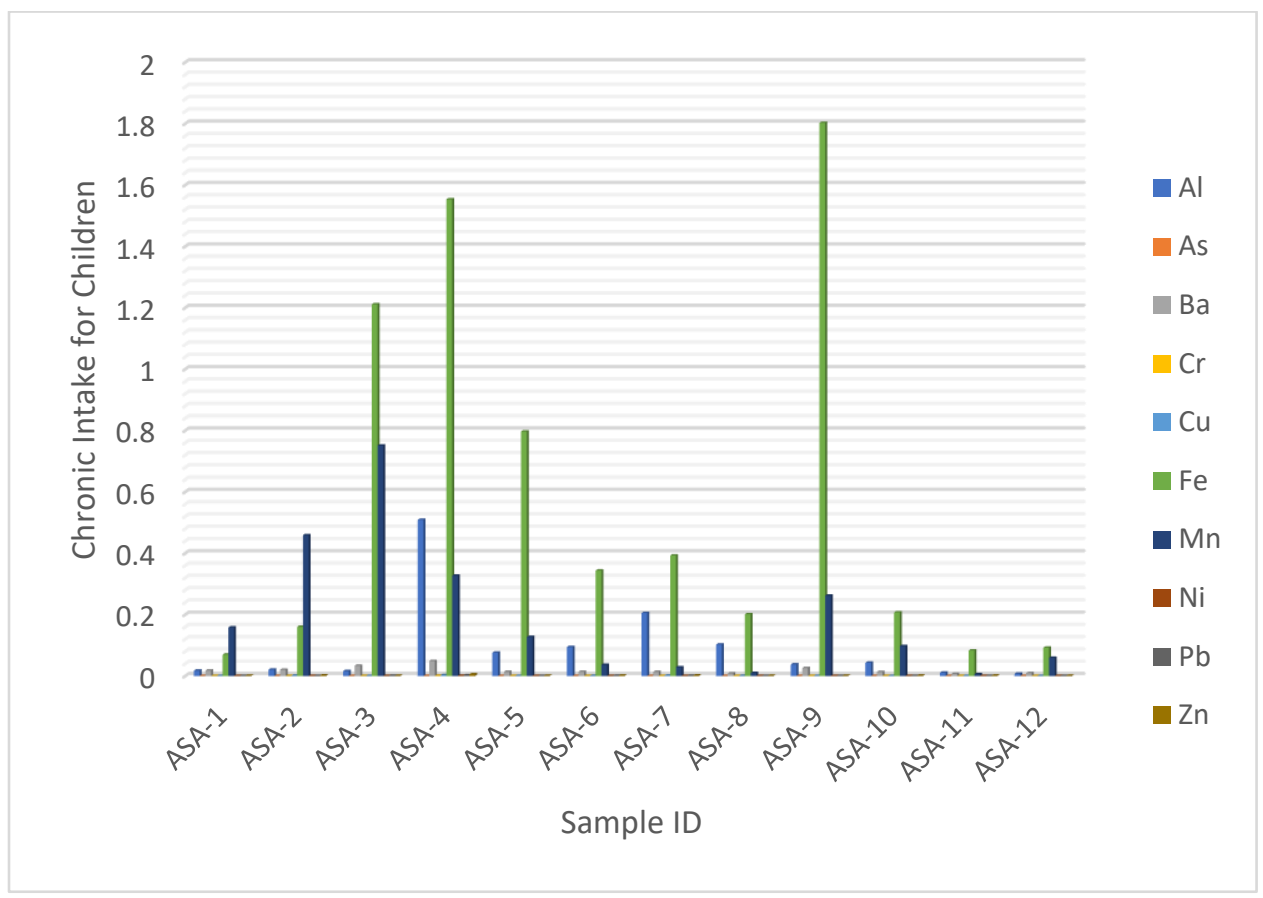

Figure 13: The Profile of Chronic Daily Intake of each of the Samples for Children

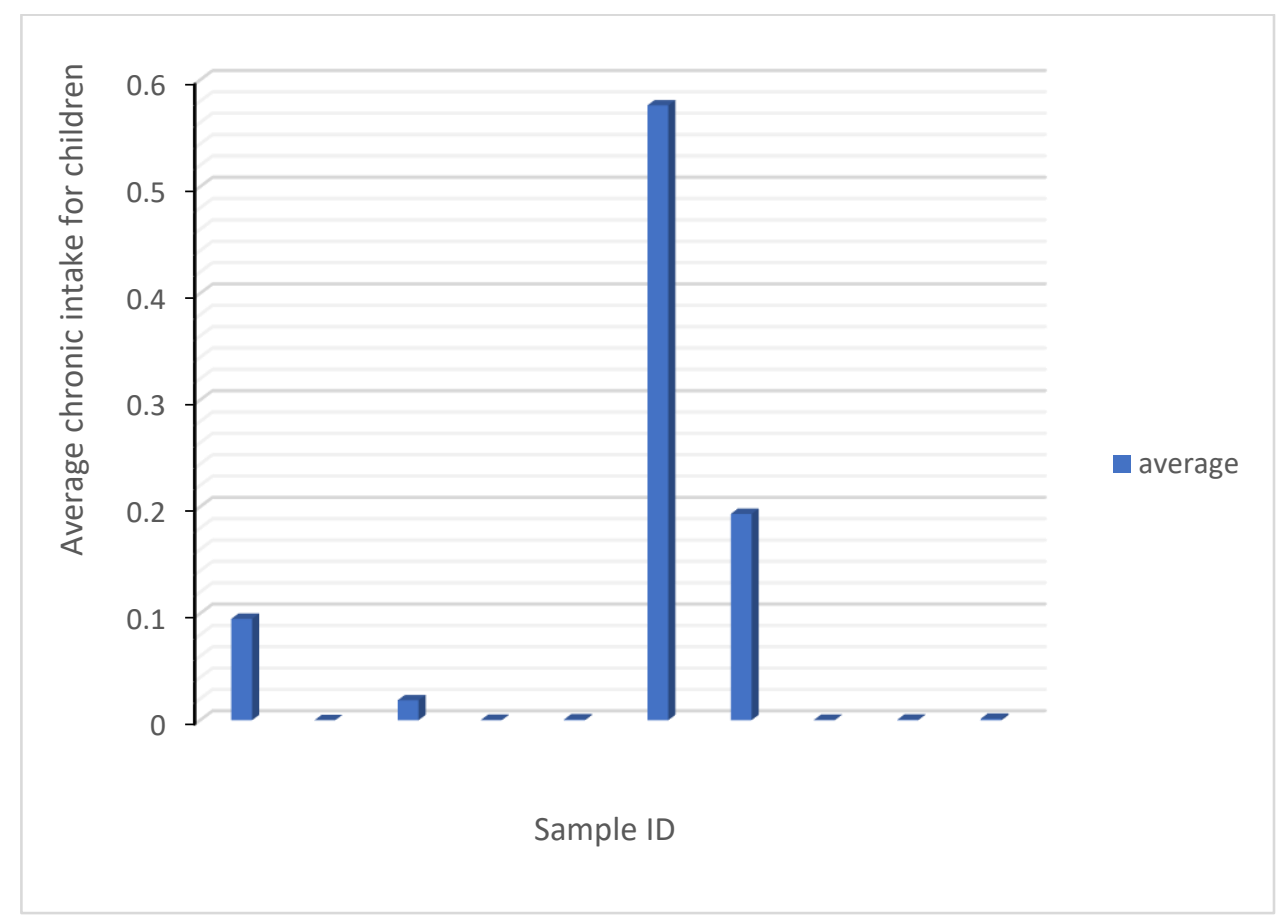

Figure 14: The Profile of Average Chronic Intake for Children 


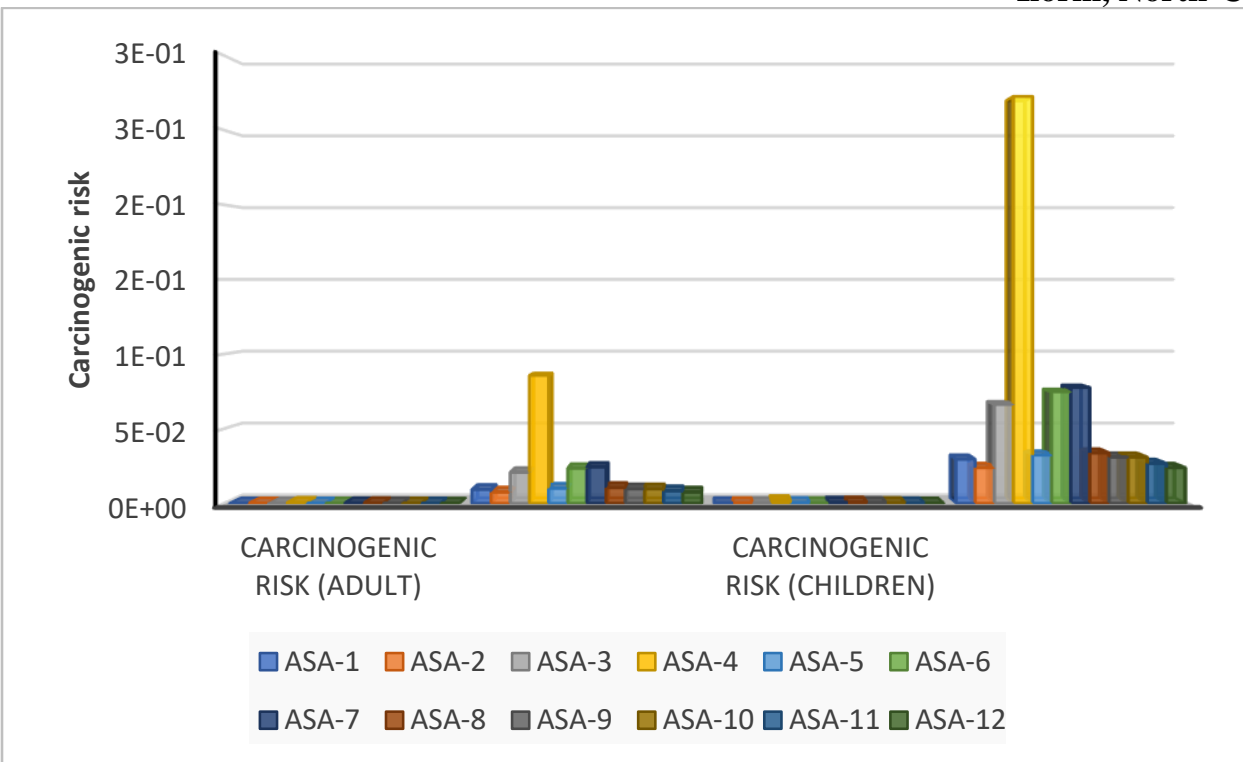

Figure 15: Profile of Carcinogenic Risk for Adults and Children

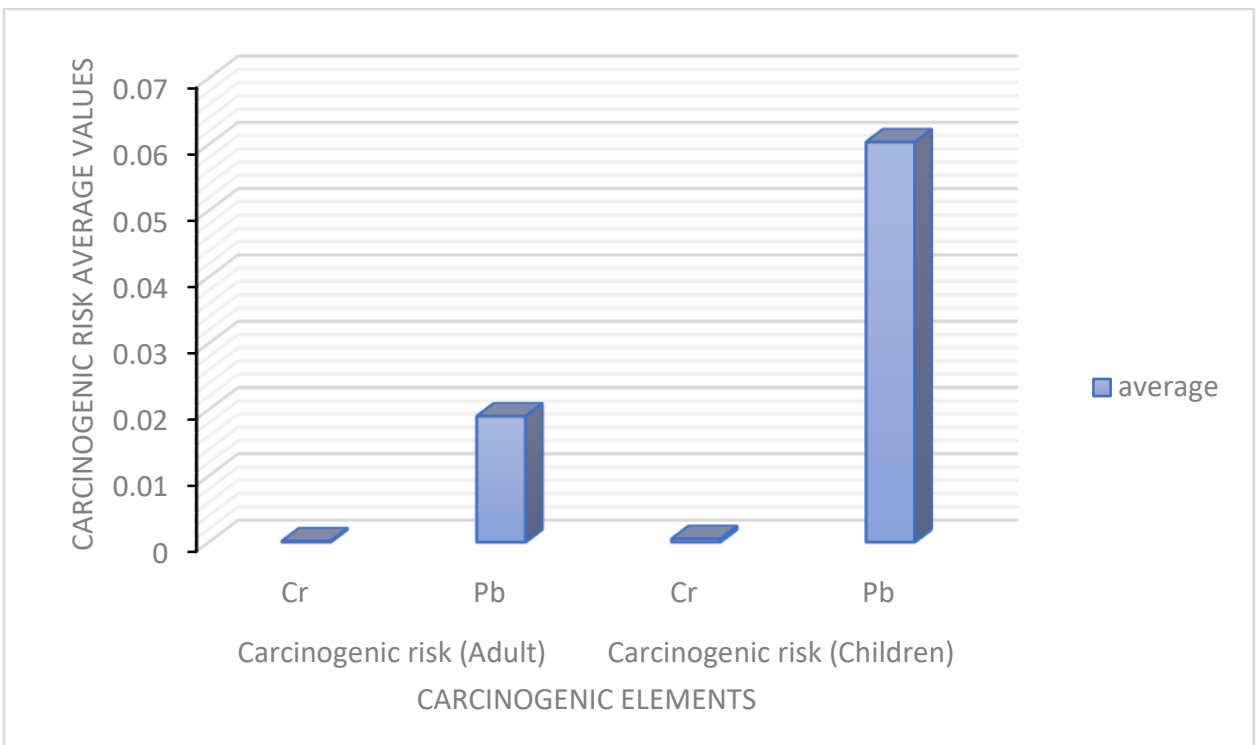

Figure 16: Profile of Average Carcinogenic risk for Adults and Children

\section{Reference}

Asare-Donkor, N. K., Boadu, T. A. and Adimado, A. A. (2016). Evaluation of groundwater and surface water quality and human risk assessment for trace metals in human settlements around the Bosomtwe Crater Lake in Ghana. Springer Plus 5(1):1812 (PubMed PMID: PMC5069212).

Atiemo, M. S., Ofosu, G. F., Mensah, H. K., Tutu, A. O., Linda-Palm, N.D.M. and Blankson, S. A. 2011. Contamination Assessment of Heavy Metals in Road Dust from Selected Roads in Accra, Ghana. Research Journal of Environmental and Earth Sciences 3(5): 473-480.

Edokpayi, J. N., Enitan, A. M., Mutileni, N. and Odiyo, J. O. (2018). Evaluation of water quality and human risk assessment due to heavy metals in groundwater around Muledane area of Vhembe District, Limpopo Province, South Africa. Chemistry Central Journal 12, 2. https://doi.org/10.1186/s13065-017-0369-y. 
LAUTECH Journal of Civil and Environmental Studies

Volume 6, Issue 1; March 2021

Ekere, N. R., Ihedioha, J. N., Eze, I. S. and Agbazue, V. E. (2014). Health risk assessment in relation to heavy metals in water sources in rural regions of south east Nigeria. 9 (6): 109-116.

Federal Survey, Nigeria (1966). Topographical Map of Nigeria, sheet 223 N.W. Ilorin.

Hynds, P.D., Thomas, M. K. and Pintar, K. D. M. (2014) Contamination of groundwater systems in the US and Canada by enteric pathogens, 1990-2013: a review and pooled- analysis. PLoS ONE 9(5): e93301.

Iqbal, J. and Shah, M. H. (2013). Health risk assessment of metals in surface water from freshwater source lakes Pakistan. Hum Ecol Risk Assess Inter. J. 19(6):1530-1543.

Joel, E. S., Maxwell, O., Adewoyin, O. O., Ehi-Eromosele,C. O., Embong, Z. and Oyawoye, F. (2018). Assessment of natural radioactivity in various commercial tiles used for building purposes in Nigeria. MethodsX, 5, 8-19. doi: 10.1016/j.mex.2017.12.002.

Li, S. Y., and Zhang, Q. F. (2010). Spatial characterization of dissolved trace elements and heavy metals in the upper Han River (China) using multivariate statistical techniques. J Hazard Mater 176(1-3):579.

Lindgren, J.F., Salo, K., Brynolf, S., Andersson, K., Svensson, E. and Zetterdahl, M. (2016). The natural environment and human impacts. Shipping and the environment. Springer, Berlin, pp 2974.

Maxwell, O., Adewoyin, O. O., Joel, E. S. and Ehi-Eromosele, C. O. (2018). Radiation exposure to dwellers due to naturally occurring radionuclides found in selected commercial building materials sold in Nigeria. Journal of Radiation Research and Applied Sciences, (in press) 11, 225-231. doi:10.1016/j. jrras.2018.01.007.

National Oceanic and Atmospheric Administration (NOAA) 2016. Available online at: https://en.wikipedia.org/wiki/Ilorin\#cite_note-NOAA-8.

Naveedullah, M. Z. H., Yu, C., Shen, H., Duan, D. and Shen, C. (2014). Concentration and human health risk assessment of selected heavy metals in surface water of the siling reservoir watershed in Zhejiang Province, China. Pol J Environ Stud 23(3):801-811.

NSDWQ, (2007). Nigerian standard for drinking water quality. Standard organization of Nigeria. http://www.unicef.org/nigeria/ng_publications_Nigerian_standard_for_drinking_water quality.pdf.

Obaje, N. G. (2009). Geological map of Nigeria showing study location.

Olasehinde, P.I., Virbka, P. and Esan, A. (1998). Preliminary Results of Hydrogeological Investigations in Ilorin area, South Western Nigeria-Quality of Hydrochemical Analysis. Water Resour. J. Natl. Assoc. Hydrogeol. 9:51-61.

Oluyide, P.O. (1979). Report on the Igbeti Marble, Oyo State of Nigeria. Report GSN, 1569, (unpublished)

Oluyide, P.O., Nwajide, C.S. and Oni, A.O. (1998). The Geology of the Ilorin Area. Bulletin No. 42 of Geological Survey of Nigeria, Published by Federal Government of Nigeria.

Omotoso, O.A., Mamodu, M.O. and Ojo, O.J. (2011). Evaluation of Geotechnical Properties of Laterite Soils, in Asa-Dam Area, Ilorin Southwestern Nigeria. World Journal of Applied Science and Technology. 3(2); 1-9.

Omotoso, O.A. and Ojo, O.J. (2017). Preliminary assessment of some selected trace elements' concentrations in Asa river water, north central Nigeria. Journal of Scientific Research, 5 (1) http://adsujsr.com/vol-5-2/ or http//www.adsujsr.com.

Omotoso, O.A., Ojo, O.J. Okebaram, U.D. and Alebiosu, M.T. (2017). Preliminary Geochemical Assessment of Asa River Sediments, North Central Nigeria. Journal of Scientific Research, 5(1):179-191. Published by Faculty of Science, Adamawa State University. Available at: http://adsujsr.com/vol-5-1/ or http//www.adsujsr.com. 


\section{Preliminary Health Risk Assessment of Asa River Water,}

Ilorin, North-Central Nigeria

Omotoso, O.A., Ojo, O.J., Olojoku, K.O., Alebiosu, M.T., Yusuf, M.A. and Kobiowu, O.O. (2018). Preliminary assessment of the quality of Asa River Water, north central Nigeria: implications for irrigation. Development Journal of science and technology research (DJOSTER), 7(2):18-35. ISSN: 2143-3275, Published by Ibrahim Badamasi Babangida University, Lapai, Niger State.

Rahaman, M.A. (1976). Review of the Basement Geology of South-Western Nigeria. Geology of Nigeria (C.A. Kogbe, ed): Elizabethan Publishing Co. Lagos.

Su, H., Kang, W., Xu, Y. and Wang, J. (2017). Assessing groundwater quality and health risks of nitrogen pollution in the Shenfu mining area of Shaanxi Province, Northwest China.Expo Health. https://doi.org/10.1007/s12403-017-0247-9.

Tijani, M.N., Okunlola, O.A. and Ikpe, E.U. (2007). A geochemical assessment of water and bottom sediments contamination of Eleyele Lake catchment, Ibadan, Southwestern Nigeria. 19(1): 105120.

USEPA, (1989). Risk assessment guidance for superfund, vol 1, human health evaluation manual (part A), Report EPA/540/1-89/002, United States Environmental Protection Agency, Washington, DC.

USEPA, (2001). Baseline human health risk assessment, Vasquez Boulevard and I-70 Superfund site. Denver, CO: U.S. Public Health Service. http://www.epa.gov/region8/ superfund/sites/VB170-Risk.pdf.

Viers, J., Dupre, B. and Gaillardet, J. (2009). Chemical composition of suspended sediments in World River: New insights from a new data base. Science of the Total Environment. 407(2):853868. www.elsevier.com/locate/scitotenv.

World Health Organization, (2006). Guidelines for drinking-water quality, addendum to volume 1: recommendations, 3rd edn. World Health Organization, Geneva.

Yuan, Y., Xiang, M., Liu, C., and Theng, B. K. G. (2017). Geochemical characteristics of heavy metal contamination induced by a sudden wastewater dis-charge from a smelter. Journal of Geochemical Exploration, 176,33-41. doi:10.1016/j. gexplo.2016.07.005. 